\title{
IFA-EO: An Improved Firefly Algorithm Hybridized With Extremal Optimization for Continuous Unconstrained Optimization Problems
}

\section{Min-Rong Chen ( $\nabla$ optmrchen@gmail.com )}

South China Normal University https://orcid.org/0000-0001-9817-0267

\section{Liu-Qing Yang}

South China Normal University

Guo-Qiang Zeng

Wenzhou University

Kang-Di Lu

Zhejiang University

Yi-Yuan Huang

South China Normal University

\section{Research Article}

Keywords: Firefly algorithm, extremal optimization, probability choice strategy, adaptive step size, continuous optimization problems

Posted Date: November 29th, 2021

DOI: https://doi.org/10.21203/rs.3.rs-190790/v1

License: (9) This work is licensed under a Creative Commons Attribution 4.0 International License. Read Full License 


\title{
IFA-EO: An improved firefly algorithm hybridized with extremal optimization
}

\section{for continuous unconstrained optimization problems}

\author{
Min-Rong Chen ${ }^{1, *}$, Liu-Qing Yang ${ }^{1}$, Guo-Qiang Zeng ${ }^{2,3 *}$, Kang-Di Lu ${ }^{4}$, Yi-Yuan Huang ${ }^{1}$ \\ ${ }^{1}$ School of Computer Science, South China Normal University, Guangzhou 510631, China \\ ${ }^{2}$ National-Local Joint Engineering Laboratory of Digitalize Electrical Design Technology, Wenzhou University, Wenzhou 325035, China \\ ${ }^{3}$ College of Cyber Security, Jinan University, Guangzhou 510632, China \\ ${ }^{4}$ Institute of Cyber Systems and Control, Zhejiang University, Hangzhou 310018, China \\ * Corresponding author, E-mail: \{mrongchen@126.com; zeng.guoqiang5@ gmail.com\}
}

\begin{abstract}
As one of the evolutionary algorithms, firefly algorithm (FA) has been widely used to solve various complex optimization problems. However, FA has significant drawbacks in slow convergence rate and is easily trapped into local optimum. To tackle these defects, this paper proposes an improved FA combined with extremal optimization (EO), named IFA-EO, where three strategies are incorporated. First, to balance the tradeoff between exploration ability and exploitation ability, we adopt a new attraction model for FA operation, which combines the full attraction model and the single attraction model through the probability choice strategy. In the single attraction model, small probability accepts the worse solution to improve the diversity of the offspring. Second, the adaptive step size is proposed based on the number of iterations to dynamically adjust the attention to the exploration model or exploitation model. Third, we combine an EO algorithm with powerful ability in local-search into FA. Experiments are tested on two group popular benchmarks including complex unimodal and multimodal functions. Our experimental results demonstrate that the proposed IFA-EO algorithm can deal with various complex optimization problems and has similar or better performance than the other eight FA variants, three EO-based algorithms, and one advanced differential evolution variant in terms of accuracy and statistical results.
\end{abstract}

Keywords: Firefly algorithm; extremal optimization; probability choice strategy; adaptive step size; continuous optimization problems.

\section{Introduction}

Most real-life engineering problems are the optimization problems in essence, such as production scheduling, investment portfolio, and vehicle routing problems, which can save resources and improve work efficiency. Recently, optimization methods for solving various optimization problems have attracted much attention in academia and engineering domain. As one of the main optimization branches, the concept of swarm intelligence was first proposed in [1]. Later, biologists and bionic algorithm experts proposed a swarm intelligence optimization algorithm based on swarm intelligence's concept. It is a bio-inspired group intelligent optimization algorithm whose idea is to draw on and simulate the functions, features, phenomena, and behaviors of natural organisms [2]. At present, the main swarm intelligence optimization algorithms include particle swarm optimization (PSO) [3], genetic algorithm (GA) [4], cuckoo search algorithm (CS) [5], bat algorithm (BA) [6], grey wolf optimization algorithm (GWO)[7], [8], flower pollination algorithm [9], firefly algorithm (FA) [10] and so on.

FA was first proposed by Yang [10]. It is a biological feature that simulates fireflies, and fireflies with high luminance attract fireflies with low brightness. Due to its simplicity in implementation and simplicity in concept, FA and its modified versions have been widely used in diverse optimization problems over the past decade [11], [12], such as demand estimation of water resources [13], path planning in uncertain environment [14], classification problems [15], image compression [16], stock forecasting [17], multimodal dynamic optimization problems [18], and RFID network planning [19]. Although FA works well for solving many optimization problems, there are still some drawbacks. In standard FA, the brighter firefly algorithm attracts all fireflies that are less bright than it. Therefore, the attractions force between fireflies is too much, resulting in oscillation and the computational time is high [20]. In order to handle such problems, many modified FAs have been introduced, such as random attraction FA [21], neighborhood attraction FA [20], switch-mode FA [22], etc. Furthermore, the standard FA uses a fixed randomization parameter during the optimization process which leads to emphasize exploration and reduces the positive attitude of exploitation [23]. 
Therefore, its convergence speed is not fast and can be fell into local optimums [22]. Many methods in the literature have been presented to copy with this problem, such as variable step size FA (VSSFA) [24], wise step strategy for FA (WSSFA) [25], FA with chaos (CFA) [26], memetic FA (MFA) [27], etc. These methods modify corresponding control parameter step size $\alpha$ or attractiveness coefficient $\beta$, which are the key control parameters to determine the performance of FA. Although these algorithms have achieved significant results, there are still some drawbacks in convergence speed and search ability. Also, the "No Free Lunch" theorem [28] illustrates that it is not possible to design one algorithm being better in solving all the optimization problems. This is one of the motivations of this work to improve the current FA for solving optimization problems.

As a novel optimization framework, extremal optimization (EO) [29], [30] is inspired by the far-from-equilibrium dynamics of self-organized criticality (SOC). EO is based on a process where the bad species is always forced to mutate according to a uniform random or power-law probability distribution. Due to its characteristics, EO has a strong ability in local search and eliminates bad components. Therefore, EO and its variations have been successfully used in a wide variety of optimization problems such as optimal controller design [31]-[33]. It is worth mentioning that EO as an ancillary technique with the exploitation ability has largely improved the performance PSO [34] and ABC [35]. A natural idea is to introduce EO in FA and test whether the performance of the improved algorithm can be enhanced. Inspired by the aforementioned descriptions, this paper proposes an IFA-EO algorithm, which hybridizes the advantages of FA and EO to reduce the computation time, improve convergence speed and prevent falling into local optimum.

Note that in [34], the authors combined PSO with EO and proposed a novel hybrid algorithm called PSO-EO. The weakness of PSO in premature convergence is compensated by introducing the EO with strong local search capability. Compared with PSO-EO, the proposed IFA-EO firstly improves the exploration ability by using single attraction model through the probability choice strategy and then combines IFA with EO. Also, in [35], the authors proposed ABC-EO, which makes full use of exploration capability of $\mathrm{ABC}$ and the exploitation capability of EO. Compared with ABC-EO, the proposed IFA-EO considers an adaptive step size based on the number of iterations to dynamically adjust the attention to the exploration model or exploitation model.

The main new contributions can be summarized below:

(1) It is a vital role in an optimization algorithm to keep the balance between exploration ability and exploitation ability. The traditional FA uses a full attraction model focusing on global search, which causes the low convergence speed. To balance between exploration ability and exploitation ability, we adopt a novel model that combines the full attraction model and the single attraction model through a probability choice strategy. In the single attraction model, small probability accepts the worse solution to improve the diversity of the offspring.

(2) The standard FA adopts a fixed step size, that is, each iteration step is equal. Different from this operation, the adaptive step size is proposed based on the number of iterations. As the number of iterations increases, the step size decreases gradually. When the time is big, the step size is close to zero.

(3) EO algorithm has only one mutation operation, which is simple and easy to implement. In this paper, we introduce EO algorithm to FA, in order to better prevent FA algorithm from trapping into the local optimum.

(4) To illustrate the performance of the IFA-EO in this paper, we use two sets of unimodal/multimodal benchmark functions to assess the performance of IFA-EO by comparing with thirteen successful swarm intelligence algorithms. We also discuss the impact of different strategies and different populations on IFA-EO. The thirteen competitors include eight variants of FA, three EO-based algorithms, and one advanced DE variant. To be more specific, the eight variants of FA are new and efficient FA (NEFA) [36], FA with adaptive control parameters (ApFA) [11], FA with neighborhood attraction (NaFA) [20], VSSFA [24], WSSFA [25], CFA [26], MFA [27], standard FA (FA) [10] and an adaptive logarithmic spiral-Levy FA (AD-IFA) [37]. Three EO-based algorithms are population-based EO (PEO) [38], PSO hybridized with EO (PSO-EO) [34], real-coded PEO algorithm with polynomial mutation (RPEO-PLM) [39]. Besides, one advanced competitor is linear population size reduction technique of success history-based adaptive differential evolution (L-SHADE) [40]. From the experimental results, we can see that IFA-EO has similar or better performance than the other thirteen optimization algorithms in terms of accuracy and statistical results. 
This paper is arranged as follows. We briefly introduce the standard FA in Section 2. Section 3 describes the proposed IFA-EO algorithm with three strategies in detail. In Section 4, the proposed IFA-EO algorithm is employed to handle two groups of unconstrained continuous benchmark functions by comparing with other competitors. Furthermore, we investigate the effects of different strategies and population size on the performance of IFA-EO. Finally, Section 5 concludes this paper and gives the future work.

\section{Firefly algorithm}

FA is a meta-heuristic algorithm derived from swarm intelligence. The basic idea of FA is to simulate the firefly flashing behavior in nature, search for a brighter one around. It gradually moves toward the better position and gathers to the brightest position, i.e., achieving the best solution. FA has a simple concept, clear process, few parameter settings, no mutation, crossover, and other complex operations, so it is easier to operate. Here, we assume that [10]:

(1) In the algorithm, the gender difference of firefly individuals is not considered, that is, all firefly individuals are considered to be of the same sex.

(2) The attraction of fireflies is based on their light intensity. Consider two fireflies, the brighter firefly will attract the weaker firefly, and the attraction will reduce as the distance between them increases.

(3) In practical application, the light intensity of firefly individuals is generally associated with objective function value, and usually objective function value is considered to be the light intensity of the firefly at that point.

Algorithm 1 gives the pseudo-code of FA [10].

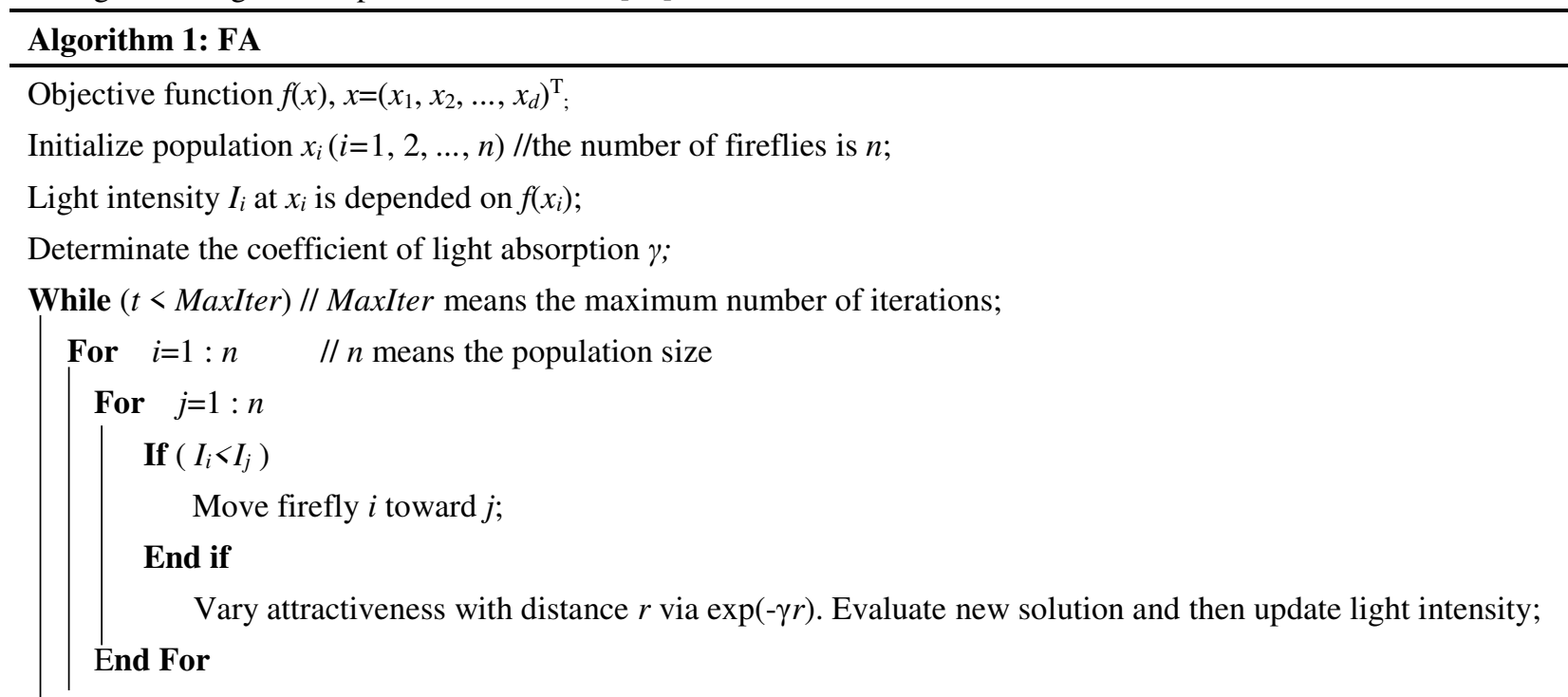

End For

Rank all the fireflies and search the current best solution $I_{g}$ found so far;

End While

The light intensity of firefly usually reduces with the increase of distance. The light intensity $I$ is given below [10]:

$$
I(r)=I_{0} e^{-\gamma r^{2}}
$$

where $I_{0}$ denotes the original light intensity of the firefly, $r$ means the distance between two fireflies. $\gamma$ is called light absorption factor.

The attractiveness $\beta$ is defined as follows [10]:

$$
\beta=\beta_{0} \mathrm{e}^{-\gamma r^{2}}
$$

where $\beta_{0}$ means the attractiveness when $r=0$. The distance between fireflies $i$ and $j$ at the spatial coordinates, i.e., $x_{i}$ and $x_{j}$, can be expressed as the Cartesian distance below [10]:

$$
r_{i, j}=\left\|x_{i}-x_{j}\right\|=\sqrt{\sum_{k=1}^{D}\left(x_{i, k}-x_{j, k}\right)^{2}}
$$

where $D$ denotes the number of dimension. $x_{i, k}$ and $x_{j, k}$ represent the $k$-th components of $x_{i}$ and $x_{j}$, respectively. In the 2-dimensional case, the distance can be described as follows [10]:

$$
r_{i, j}=\sqrt{\left(x_{i}-x_{j}\right)^{2}+\left(y_{i}-y_{j}\right)^{2}}
$$

The movement of a firefly $i$ attracting to another more attractive firefly $j$ is defined as the following equation [10]: 


$$
x_{i}^{(t+1)}=x_{i}^{t}+\beta_{0} e^{-\gamma r_{i, j}^{2}}\left(x_{j}-x_{i}\right)+\alpha \varepsilon_{i}
$$

where $\alpha$ is step size, and $\varepsilon_{i}$ means a vector of random numbers from a Gaussian distribution or uniform distribution. $\alpha$ ranges from 0 to $1, \varepsilon_{i}$ is usually replaced by (rand -0.5) where rand is a random number uniformly distributed in $[0,1]$.

\section{The proposed approach}

The traditional FA has a slow convergence speed, premature convergence, and easily falling in the local optimum, which leads to low solution accuracy [44]-[46]. To deal with these problems, this study presents an improved FA called IFA-EO. In IFA-EO, three improvement strategies are adopted. Firstly, a probability choice strategy and small probability acceptance of the worse solution are used to improve the diversity of offspring. Secondly, adaptive step size is adopted to keep the balance between exploration ability and exploitation ability. Thirdly, extremal optimization (EO) [34] is suggested to enhance the local search capability of FA.

\subsection{An attraction with probability choice strategy}

Owing to the merits such as simple concept, clear process, no complexity operations, FA has attracted much attention from many scholars. Thus, many modified versions of FA have been presented in recent decades. These versions of FA are essential to strengthen the search ability and improve the accuracy of FA. On the one hand, if we pay too much attention to exploration, the convergence speed of the algorithm will slow at the end of the searching phase; on the other hand, if too much attention is paid to the exploitation process, the algorithm will easily prematurely converge. Therefore, how to balance exploration ability and exploitation ability is very important. In general, at the beginning of optimization, exploration operation is more important because we need quickly and roughly search the entire search space to find the most potential areas. To tackle this problem, we propose an attraction model with a probability choice strategy. The attraction model is described as follows.

The probability is calculated by using Eq. (6) [47]:

$$
P=P_{\text {min }}+\left(P_{\text {max }}-P_{\text {min }}\right) \times e^{1-\frac{\text { MaxIter }}{\text { MaxIter }-t+1}}
$$

where $P_{\min }$ is the minimum value of attraction probability, $P_{\max }$ means the maximum value of attraction probability, MaxIter means the maximum iteration number, and $t$ denotes the current iteration number. $P_{\max }, P_{\min }$ range from 0 to 1 , $P_{\max }, P_{\min }$ usually set as $0.9,0.05$, respectively. As the number of iterations increases, $P$ will gradually decrease from $P_{\max }$ to $P_{\min }$.

In each iteration, a random number $q$ is obtained between 0 and 1 . If $q<P$, we adopt a full attraction model [10], as shown in Fig. 1. We can see that the $i$ th firefly is attracted to other fireflies. It is beneficial to perform exploration at an early stage. Otherwise, if $q>P$, single attraction model is used, as shown in Fig. 2. In the single attraction model, the $i$ th firefly is attracted to only one neighbor $i$-1th firefly, which is a better solution than the $i$ th firefly. Emphasize that $x_{i-1}$ in Fig. 2 is $i$-1 th firefly sorted according to the descending order of light intensity. It makes the convergence speed very fast at the later period.

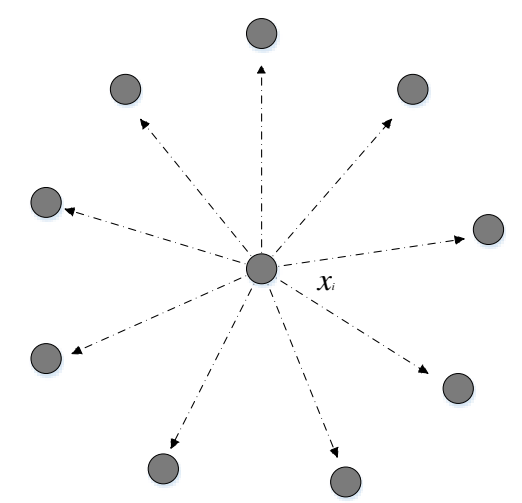

Fig. 1. Full attraction model [10]

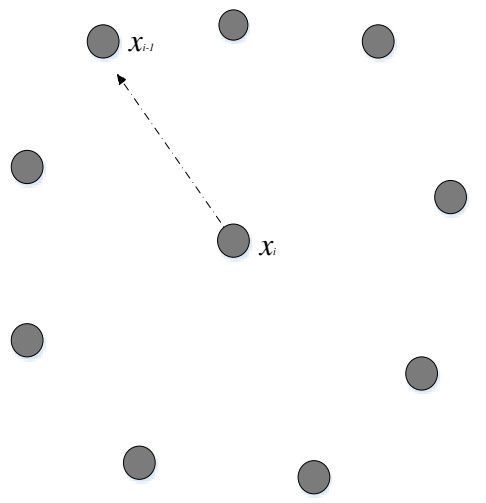

Fig. 2. Single attraction model

In the single model, it may only focus on local search, leading to premature convergence. In order to jump out of local optimum, inspired by the basic idea of simulated annealing, we accept some worse solutions with a small 
probability in the single model, that is, fireflies have chances to move towards darker fireflies to avoid falling into local optimum.

\subsection{Adaptive step size}

In the standard FA, using a fixed step size is not conducive to balancing exploration and exploitation. When the step size is set too large, the search at the later stage may skip the optimal solutions, otherwise, the convergence speed is too slow. The steps size of FA should be tuned according to actual conditions. To solve this problem, Yu et al. [24] proposed VSSFA and WSSFA, respectively. Furthermore, in [11], Wang et al. discussed the relationship between the rate of convergence and step size $\alpha$. If FA is convergent, the parameter $\alpha$ will satisfy the condition as follows [11]:

$$
\lim _{t \rightarrow \infty} \alpha=0
$$

Inspired by Eq. (7), we propose an adaptive step size $\alpha$ by using a nonlinear equation. The ability to balance exploration and exploitation will be beneficial, and it should also focus on its current iterations. The adaptive step size $\alpha$ is updated as follows:

$$
\alpha(t+1)=\theta^{\left(\frac{t}{\lambda \cdot \text { Maxlter }}\right)} \cdot \alpha(t)
$$

where $\alpha(t)$ is the value of $\alpha$ at the current iteration, $\alpha$ ranges from 0 to 1 . Dynamic step size $\alpha$ convergence curve is given in Fig. 3. From Fig. 3, it can be seen that the step is large at an early phase, and then reduces when iteration increases. It is evident that our adaptive step satisfies the convergence condition Eq. (7) [11].

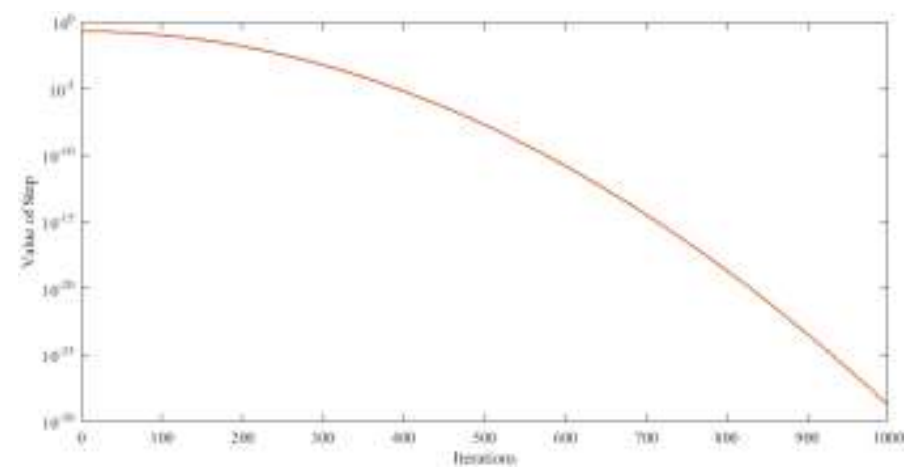

Fig. 3. The change of step size in 1000 iteration

\begin{tabular}{|c|c|c|c|c|c|c|c|c|}
\hline \multirow{2}{*}{ Function } & \multicolumn{2}{|c|}{$\theta=200 / 1013$} & \multicolumn{2}{|c|}{$\theta=500 / 1013$} & \multicolumn{2}{|c|}{$\theta=800 / 1013$} & \multicolumn{2}{|c|}{$\theta=1000 / 1013$} \\
\hline & $f_{\mathrm{m}}$ & SD & $f_{\mathrm{m}}$ & SD & $f_{\mathrm{m}}$ & $\mathrm{SD}$ & $f_{\mathrm{m}}$ & $\mathrm{SD}$ \\
\hline$f_{1}$ & $5.84 \mathrm{E}-06$ & $1.12 \mathrm{E}-05$ & $5.37 \mathrm{E}-10$ & 2.33E-09 & $2.27 \mathrm{E}-32$ & $6.91 \mathrm{E}-32$ & 1.10E-108 & 5.73E-108 \\
\hline$f_{2}$ & $7.57 \mathrm{E}-03$ & $5.81 \mathrm{E}-03$ & $6.63 \mathrm{E}-04$ & $6.38 \mathrm{E}-04$ & $4.88 \mathrm{E}-05$ & 7.95E-05 & 3.11E-42 & $9.18 \mathrm{E}-42$ \\
\hline$f_{3}$ & $8.43 \mathrm{E}+02$ & $3.92 \mathrm{E}+02$ & $3.86 \mathrm{E}+02$ & $1.61 \mathrm{E}+02$ & $5.32 \mathrm{E}+01$ & $4.23 \mathrm{E}+01$ & 4.93E-34 & 2.59E-33 \\
\hline$f_{4}$ & $5.98 \mathrm{E}-01$ & $5.01 \mathrm{E}-01$ & $1.42 \mathrm{E}-02$ & $1.00 \mathrm{E}-02$ & $9.43 \mathrm{E}-04$ & $1.14 \mathrm{E}-03$ & 7.39E-35 & 3.81E-34 \\
\hline$f_{5}$ & $4.45 \mathrm{E}+01$ & $2.73 \mathrm{E}+01$ & $4.48 \mathrm{E}+01$ & $2.73 \mathrm{E}+01$ & $4.16 \mathrm{E}+01$ & $2.69 \mathrm{E}+01$ & $2.80 \mathrm{E}+01$ & $1.58 \mathrm{E}+01$ \\
\hline$f_{6}$ & $0.00 \mathrm{E}+00$ & $0.00 \mathrm{E}+00$ & $0.00 \mathrm{E}+00$ & $0.00 \mathrm{E}+00$ & $0.00 \mathrm{E}+00$ & $0.00 \mathrm{E}+00$ & $0.00 \mathrm{E}+00$ & $0.00 \mathrm{E}+00$ \\
\hline$f_{7}$ & $5.81 \mathrm{E}-01$ & $2.86 \mathrm{E}-01$ & $5.56 \mathrm{E}-01$ & $2.65 E-01$ & $5.45 \mathrm{E}-01$ & $2.97 \mathrm{E}-01$ & 4.45E-01 & $2.86 \mathrm{E}-01$ \\
\hline$f_{8}$ & $6.00 \mathrm{E}+03$ & $8.46 \mathrm{E}+02$ & $5.33 \mathrm{E}+03$ & $6.30 \mathrm{E}+02$ & $4.80 \mathrm{E}+03$ & $8.15 E+02$ & $3.56 \mathrm{E}+03$ & $6.95 \mathrm{E}+02$ \\
\hline$f_{9}$ & 1.08E-01 & 1.01E-01 & $1.65 \mathrm{E}-01$ & 2.44E-01 & $1.42 \mathrm{E}-01$ & $1.49 \mathrm{E}-01$ & $1.33 \mathrm{E}+00$ & $1.01 \mathrm{E}+00$ \\
\hline$f_{10}$ & $5.22 \mathrm{E}-03$ & $1.22 \mathrm{E}-02$ & $6.32 \mathrm{E}-07$ & $1.05 \mathrm{E}-06$ & $5.10 \mathrm{E}-14$ & $1.95 \mathrm{E}-14$ & $2.76 \mathrm{E}-14$ & 7.13E-15 \\
\hline$f_{11}$ & $4.87 \mathrm{E}-03$ & $6.87 \mathrm{E}-03$ & $2.05 \mathrm{E}-03$ & $4.89 \mathrm{E}-03$ & $1.31 \mathrm{E}-03$ & 2.97E-03 & 2.59E-17 & $6.20 \mathrm{E}-17$ \\
\hline$f_{12}$ & $7.10 \mathrm{E}-03$ & $2.59 \mathrm{E}-02$ & 7.02E-08 & $1.87 \mathrm{E}-07$ & $7.72 \mathrm{E}-16$ & $2.87 \mathrm{E}-15$ & $1.64 \mathrm{E}-32$ & $2.20 \mathrm{E}-33$ \\
\hline$f_{13}$ & $9.09 \mathrm{E}-04$ & $2.81 \mathrm{E}-03$ & $6.09 \mathrm{E}-04$ & $2.08 \mathrm{E}-03$ & $2.23 \mathrm{E}-15$ & $1.04 \mathrm{E}-14$ & 3.13E-32 & $9.70 E-33$ \\
\hline
\end{tabular}

Table 1: Comparative performance for test functions $f_{1}-f_{13}$ with different $\theta$ values. 
From Eq. (8), we can see that the size of $\theta$ and $\lambda$ are important factors for $\alpha$. The empirical value of $\lambda$ is 0.1 . In Eq. (8), if $\lim _{t \rightarrow \infty} \alpha=0$, the parameter $\theta$ should satisfy $0<\theta<1$. Different $\theta$ values will obtain different results. We adopt thirteen well-known benchmark functions from the literature [20] to test different $\theta$ values. In this experiment, MaxIter and population size are set as 5000 and 20, respectively. The parameter $\theta$ is set as 200/1013, 500/1013, 800/1013, and 1000/1013, respectively. Table 1 lists the compared results for different $\theta$ values, where $f_{\mathrm{m}}$ denotes the mean of best function values and SD represents standard deviation. From Table 1, we can see that $\theta=1000 / 1013$ obtains better solutions than those with other $\theta$ values on thirteen functions. Thus, $\theta=1000 / 1013$ is considered in all the following experiments.

\subsection{Hybridized with Extremal Optimization (EO) algorithm}

Compared with other swarm intelligent algorithms, EO algorithm exist form is not a population but exists as a single individual. An individual can be made up of many components. For instance, if $X=\left(x_{1}, x_{2}, x_{3}\right)$, then $x_{1}, x_{2}$, and $x_{3}$ are named components of $X$. Additionally, the basic EO does not need to adjust any parameters when searching, and there is only a mutation operator. Although FA offers fast exploration and exploitation, the exploitation ability of FA can be further strengthened by EO with the merits in local search ability. However, if EO is added into FA in every generation, it will slow down the convergence speed of FA and needs more computational cost. Thus, EO is introduced into FA at $I N V$-iteration intervals. The parameter $I N V$ means the obtained global optimal solution is unchanged for $I N V$-iterations. For a simple benchmark function, the INV range can be larger, with a range of 50-100 being more appropriate. For complex benchmark functions, the range of $I N V$ should be small, and the value range should be between 1 and 50 [34].

Due to only mutation operator in EO, it plays a vital role in the whole procedure. In this study, we use the hybrid Gaussian-Cauchy mutation operator [34]. The EO introduced in FA for a minimization problem is given in Algorithm 2 [34] and Fig. 4 gives the corresponding process.
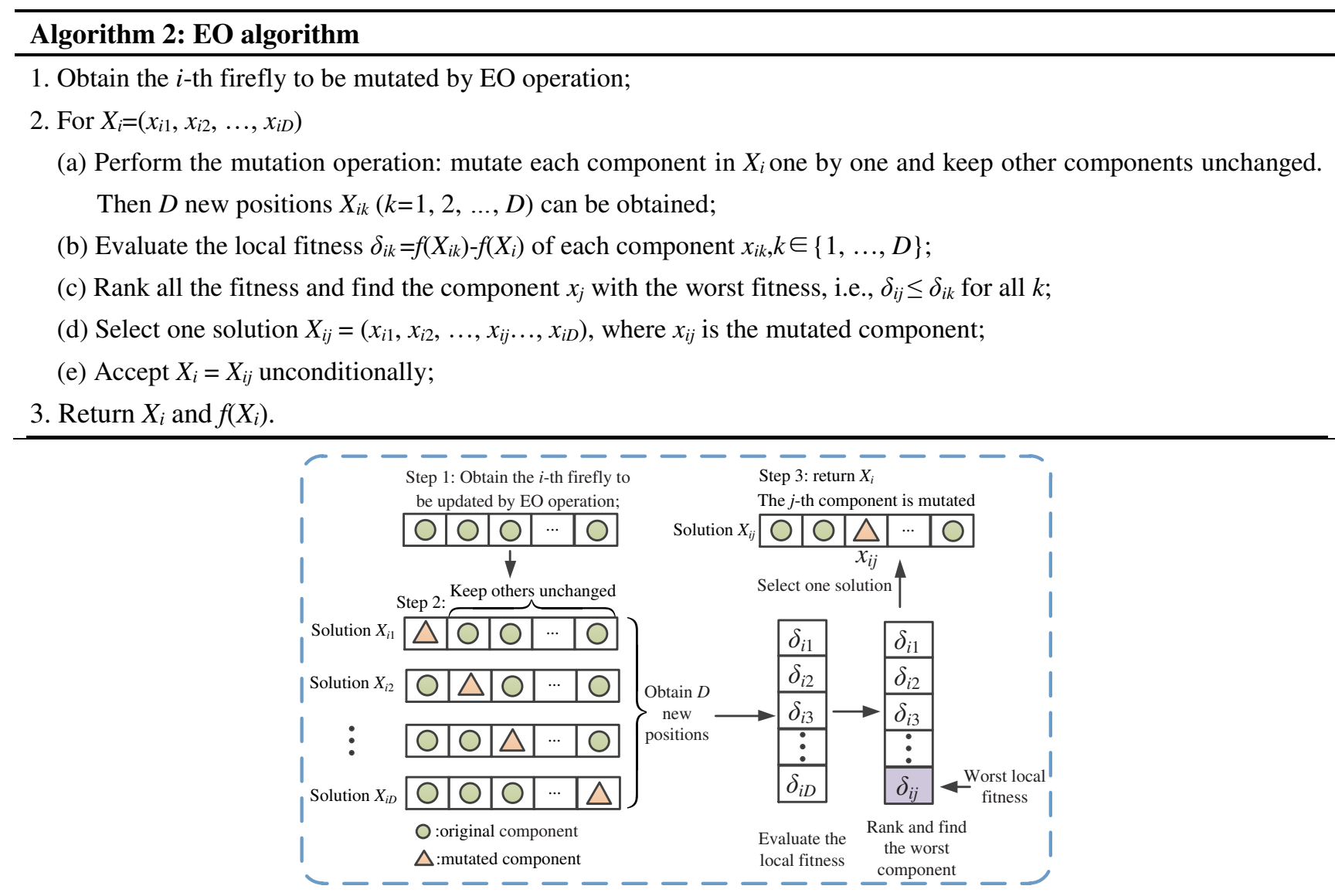

Fig. 4 The process of EO 


\subsection{Framework of IFA-EO}

The proposed IFA-EO uses MFA's movement formula [27]. In memetic FA, the movement formula is redefined. The movement formula is defined as follows [27].

$$
\begin{gathered}
x_{i}=x_{i}+\beta e^{-\gamma r_{i, j}{ }^{2}}\left(x_{j}-x_{i}\right)+\alpha s_{d} \varepsilon_{i} \\
\beta=\beta_{\text {min }}+\left(\beta_{0}-\beta_{\text {min }}\right) e^{-\gamma r_{i j}^{2}}
\end{gathered}
$$

where $\beta_{\min }$ means the minimum value of $\beta$, and $s_{d}$ means the length scale of each designed variable. The values of $\beta_{\text {min }}$ and $\beta_{0}$ range from 0 to 1 . From Eq. (10), the range of attractiveness $\beta$ is $\beta_{\min }$ to $\beta_{0}$.

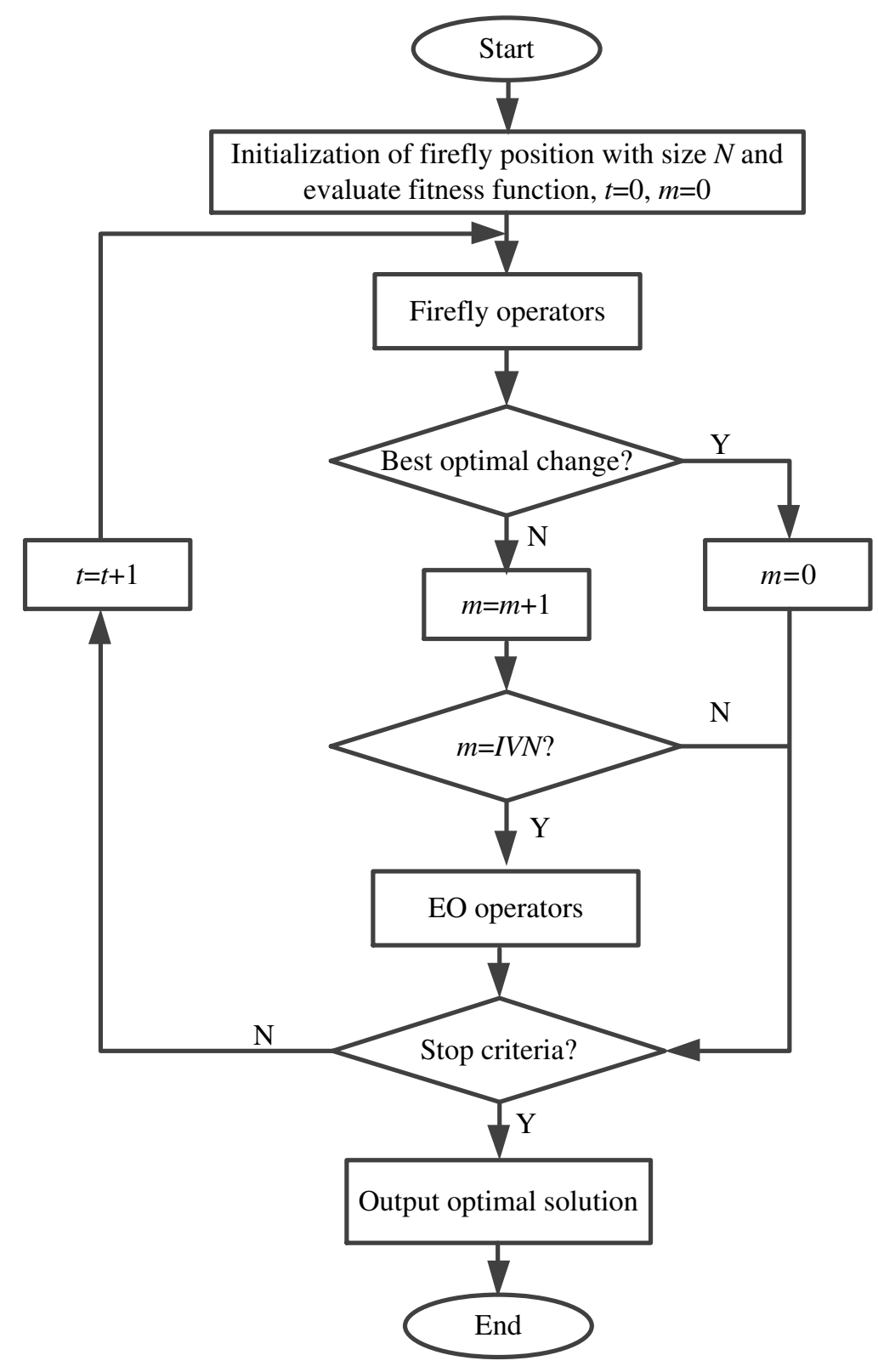

Fig. 5. Flowchart of IFA-EO algorithm.

The IFA-EO algorithm flowchart is illustrated in Fig. 5, and corresponding framework of IFA-EO is described in Algorithm 3. 


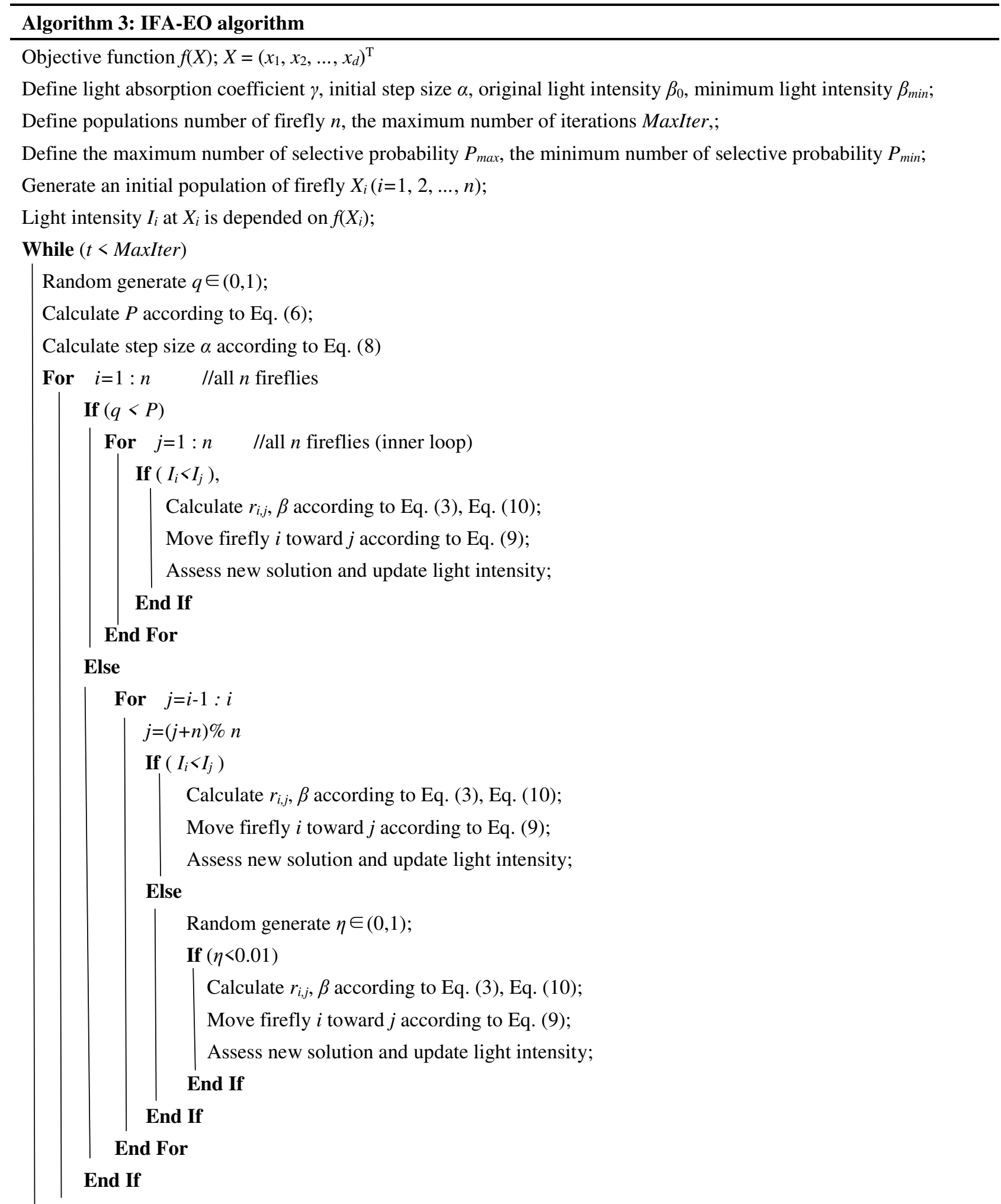

\section{End For}

If the best solution is unchanged for $I N V$-generations, then EO procedure is introduced Rank all the fireflies and find the current best solution $I_{g}$ found so far.

\section{End While}

\subsection{Computational complexity of our algorithms}

According to the pseudo-code of FA [10], it can be found that the computational complexity of FA is $O\left(N^{2}\right.$ MaxIter $)$. Compared to other intelligent optimization algorithms, including PSO, ABC, and BA, standard FA has higher complexity. In one iteration, the algorithm complexity of PSO, ABC, and BA are both $O(N)$. Since the attracting model of our algorithm adopts the probability choice strategy and introduces the EO algorithm, our algorithm complexity is divided into the following situations. We only study the computational complexity at one iteration of IFA-EO. 
- When using a single attraction mode and not introducing EO, the algorithm complexity is $O(N)$, where $N$ means the population size, and $D$ means the number of dimensions.

- When using a single attraction mode and introducing EO, the algorithm complexity is $O(N D)$.

- When using a full attraction mode and not introducing EO, the algorithm complexity is $O\left(N^{2}\right)$.

- When using a full attraction mode and introducing EO, the algorithm complexity is $O\left(N^{2} D\right)$.

From the above analysis, we can see the complexity of IFA-EO is $O(N)$ in the best case and $O\left(N^{2} D\right)$ in the worst case. From the above analysis, we can summarize as follows, compared to the FA, in the worst case, IFA-EO has a slightly higher computational complexity. But in the best case, the computational complexity of IFA-EO is very low.

Remark 1: Computational complexity is also important in the evolutionary algorithm domain. To achieve a reasonable level of optimization, the smaller computational complexity of evolutionary algorithm will save more computational resources. Also, there are many works in discussing in computational complexity of evolutionary algorithms, e.g., Ref.[48].

\section{Experimental results and discussion}

\subsection{Summary of experimental conditions}

Table 2: Summary of experimental conditions

\begin{tabular}{|c|c|c|c|c|c|c|}
\hline Experiment & Test problem & Dimension & Algorithm & Population size & MaxIter/MaxFEs & Number of runs \\
\hline Exp. 1 & $f_{1}-f_{13}$ & 30 & $\begin{array}{c}\text { IFA-EO } \\
\text { ApFA [11] } \\
\text { NaFA [20] } \\
\text { VSSFA [24] } \\
\text { WSSFA [25] } \\
\text { CFA [26] } \\
\text { MFA [27] } \\
\text { FA [10] }\end{array}$ & 20 & $5.0 \mathrm{E}+05$ & 30 \\
\hline Exp. 2 & $h_{1}-h_{8}$ & 100 & $\begin{array}{c}\text { IFA-EO } \\
\text { RPEO-PLM [39] } \\
\text { PSO-EO [34] } \\
\text { PEO [38] }\end{array}$ & 30 & $\begin{array}{c}\text { MaxIter } \\
5000 \text { (IFA-EO) } \\
12000 \text { (Others) }\end{array}$ & 30 \\
\hline Exp. 3 & $f_{1}-f_{13}$ & 50 & $\begin{array}{c}\text { IFA-EO } \\
\text { AD-IFA[37] } \\
\text { L-SHADE [40] }\end{array}$ & 20 & $3.0 \mathrm{E}+05$ & 30 \\
\hline Exp. 4 & $f_{1}-f_{13}$ & 30 & $\begin{array}{c}\text { FA } \\
\text { FA-A } \\
\text { FA-EO } \\
\text { FA-PA } \\
\text { IFA-EO }\end{array}$ & 20 & $5.0 \mathrm{E}+05$ & 30 \\
\hline Exp. 5 & $f_{1}-f_{13}$ & 30 & IFA-EO & $\begin{array}{l}10 \\
20 \\
30 \\
40\end{array}$ & $5.0 \mathrm{E}+05$ & 30 \\
\hline
\end{tabular}

In this section, we illustrate the performance of the proposed IFA-EO algorithm according to five experiments. Table 2 presents conditions of each experiment including dimension, the competitors, population size, the maximum number of fitness evaluations, and the number of runs. Five experiments are designed for different purposes. To be more specific, in the first experiment (Exp. 1), the aim is to verify that the proposed IFA-EO as a kind of FA variant can 
realize better performance than other FA variants. Thus, seven FA variants, i.e., ApFA [11], NaFA [20], VSSFA [24], WSSFA [25], CFA [26], MFA [27] and FA [10] are viewed as the competitors. Considering the IFA-EO is efficiently using EO mechanism, EO-based algorithms are needed to compare. Here, we choose three recently related methods i.e., PEO [38], PSO-EO [34], and RPEO-PLM [39] as comparisons in the second experiment (Exp. 2). To further prove the performance of IFA-EO, the third experiment is devoted to comparing IFA-EO algorithm with other two well-recognized algorithms. These two competitors are AD-IFA [37] and L-HSADE [40]. In the fourth experiment (Exp. 4), FA with different modified strategies is shown to demonstrate the performance of different strategies. In addition, to investigate the impact of population size on the performance of IFA-EO, we design the fifth experiment (Exp. 5).

It is worth mentioning that different adjustable parameters may influence the performance of IFA-EO. Here, the related parameters are determined by the trial-and-error method. In all the following experiments, the initial parameters $\lambda, \alpha, \gamma, \beta_{0}$, and $\beta_{\min }$ are set as $0.1,0.2,1.0,1.0$ and 0.2 , respectively. The minimum and maximum attraction probability $P_{\min ,} P_{\max }$ are set to 0.05 and 0.8 , respectively. The considered functions are all minimized problems. Experiments 1-5 of IFA-EO are performed in JAVA software on a $3.20 \mathrm{GHz}$ computer with processor i5-6500U and 8 GB RAM.

\subsection{Exp. 1: Comparison with FA variants}

Table 3: Benchmark functions $f_{1}-f_{13}$ with $D=30$.

\begin{tabular}{|c|c|c|c|}
\hline Problem & Function expression & Search space & Global minimum \\
\hline$f_{1}$ & $f_{1}(x)=\sum_{i=1}^{D} x_{i}^{2}$ & {$[-100,100]$} & 0 \\
\hline$f_{2}$ & $f_{2}(x)=\sum_{i=1}^{D}\left|x_{i}\right|+\prod_{i=1}^{D}\left|x_{i}\right|$ & {$[-10,10]$} & 0 \\
\hline$f_{3}$ & $f_{3}(x)=\sum_{i=1}^{D}\left(\sum_{j=1}^{i} x_{j}\right)^{2}$ & {$[-100,100]$} & 0 \\
\hline$f_{4}$ & $f_{4}(x)=\max \left\{\left|x_{i}\right|, 1 \leq i \leq D\right\}$ & {$[-100,100]$} & 0 \\
\hline$f_{5}$ & $f_{5}(x)=\sum_{i=1}^{D-1}\left(100\left(x_{i}^{2}-x_{i+1}\right)^{2}+\left(x_{i}-1\right)^{2}\right)$ & {$[-30,30]$} & 0 \\
\hline$f_{6}$ & $f_{6}(x)=\sum^{D}\left(\left\lfloor x_{i}+0.5\right\rfloor\right)^{2}$ & {$[-100,100]$} & 0 \\
\hline$f_{7}$ & $f_{7}(x)=\sum_{i=1}^{D} i x_{i}^{4}+$ random $[0,1)$ & {$[-1.28,1.28]$} & 0 \\
\hline$f_{8}$ & $f_{8}(x)=418.9829 \cdot D-\sum_{i=1}^{D} x_{i} \sin \left(\sqrt{\left|x_{i}\right|}\right)$ & {$[-500,500]$} & 0 \\
\hline$f_{9}$ & $f_{9}(x)=\sum_{i=1}^{D}\left[x_{i}^{2}-10 \cos 2 \pi x_{i}+10\right]$ & {$[-5.12,5.12]$} & 0 \\
\hline$f_{10}$ & $f_{10}(x)=-20 \exp \left(-0.2 \sqrt{\frac{1}{D} \sum_{i=1}^{D} x_{i}}\right)-\exp \left(\frac{1}{D} \sum_{i=1}^{D} \cos \left(2 \pi x_{i}\right)\right)+20+e$ & {$[-32,32]$} & 0 \\
\hline$f_{11}$ & $f_{11}(x)=\sum_{i=1}^{D} \frac{x_{i}^{2}}{4000}-\prod_{i=1}^{D} \cos \left(\frac{x_{i}}{\sqrt{i}}\right)+1$ & {$[-600,600]$} & 0 \\
\hline$f_{12}$ & $\begin{aligned} f_{12} & =\frac{\pi}{D}\left\{\left(y_{D}-1\right)^{2}+10 \sin ^{2}\left(\pi y_{1}\right)+\sum_{i=1}^{D-1}\left(y_{i}-1\right)^{2}\left[1+10 \sin ^{2}\left(\pi y_{i+1}\right)\right]\right\} \\
& +\sum_{i=1}^{D} u\left(x_{i}, 10,100,4\right), \quad y_{i}=1+\frac{x_{i}+1}{4} \\
& u\left(x_{i}, a, k, m\right)= \begin{cases}k\left(x_{i}-a\right)^{m}, & x_{i}>a \\
0, & -a \leq x_{i} \leq a \\
k\left(-x_{i}-a\right)^{m}, & x_{i}<-a\end{cases} \end{aligned}$ & {$[-50,50]$} & 0 \\
\hline
\end{tabular}




$$
\begin{aligned}
f_{13} & =0.1\left\{\sin ^{2}\left(3 \pi x_{1}\right)+\sum_{i=1}^{D-1}\left(x_{i}-1\right)^{2}\left[1+\sin ^{2}\left(3 \pi x_{i+1}\right)\right]\right. \\
& \left.+\left(x_{D}-1\right)^{2}\left[1+\sin ^{2}\left(2 \pi x_{D}\right)\right]\right\}+\sum_{i=1}^{D} u\left(x_{i}, 5,100,4\right)
\end{aligned}
$$

This group of well-known benchmark functions shown in Table 3 is selected from the literature [20]. For these test functions in Table 3 , since the $f_{1}, f_{2}, f_{4}, f_{6}, f_{10}, f_{12}, f_{13}$ are simple test functions, the firefly procedures can find a better solution, so when the optimal solution does not change for 500 generations, we introduce EO. For $f_{3}$, the value of $I N V$ is 100. For complex test functions, the $f_{11}$, we set $I N V$ as 10 and the $f_{7}, I N V=50$. Since the $f_{5}, f_{8}, f_{9}$ function is easy to trap into the local optimums, we introduce EO when the optimal solution does not change in five generations. Recently, some FA variants, e.g., ApFA [11], NaFA [20], VSSFA [24], WSSFA [25], CFA [26], MFA [27] and FA [10] have been proposed. Due to the improvement on standard FA, these FA variants have been proved their performance through various optimization problems. The main framework of IFA-EO algorithm is FA, which can be viewed as another FA variant. Thus, it is necessary to compare with the above seven FA variants to verify that IFA-EO is another efficient FA variant. Table 4 presents average results $f_{\mathrm{m}}$ of these eight FA variants for thirteen benchmark test functions on 30 run times. The experimental results of algorithms FA, VSSFA, WSSFA, MFA, CFA, and NaFA are excerpted from the literature [20]. The results of ApFA are taken from reference [11]. Note that bold values in the table of this paper mean the best unless otherwise stated. For Table 5, it can be seen that IFA-EO algorithm performs the best in 8 out of 13 test functions $f_{1}-f_{13}$, the same as $f_{6}$ for MFA, CFA, NaFA, and ApFA, and the same as NafA for function $f_{11}$. For some low-dimensional problems, VSSFA and WSSFA have good performance [24], [25], but they fail to obtain well solutions compared to other FA variants on 30-dimensional problems. The comparison results of IFA-EO and the other seven FA variants are denoted by $w / t / l$, which indicates that IFA-EO has better performance in $w$ functions, similar performance in $t$ functions, and worse performance in $l$ functions. Thus, compared to the other seven competitors, IFA-EO can achieve almost the same or better performance on the considered functions $f_{1}-f_{13}$.

In addition, we use two non-parametric statistic tests [49], i.e., Friedman test and Quade test to further compare all eight FA variants and the Bonfreeoni-Dunn method is selected as the post-hoc test performed by the KEEL [50]. Table 5 presents ranks, statistics, and $p$-values achieved by two non-parametric statistic tests for IFA-EO and other seven FAs. It is obvious that IFA-EO ranks the first in Quade tests with a level of significance $\alpha=0.05$. NaFA and IFA-EO obtains

\begin{tabular}{|c|c|c|c|c|c|c|c|c|}
\hline Function & $\mathrm{FA}[10]$ & MFA[27] & CFA[26] & WSSFA[25] & VSSFA[24] & $\mathrm{NaFA}[20]$ & ApFA[11] & IFA-EO \\
\hline$f_{1}$ & $5.67 \mathrm{E}-02$ & 4.07E-06 & $3.27 \mathrm{E}-06$ & $6.34 \mathrm{E}+04$ & $5.84 \mathrm{E}+04$ & $4.43 \mathrm{E}-29$ & $2.02 \mathrm{E}-44$ & 1.10E-108 \\
\hline$f_{2}$ & $1.00 \mathrm{E}+00$ & $9.16 \mathrm{E}-04$ & $8.06 \mathrm{E}-04$ & $1.35 \mathrm{E}+02$ & $1.13 \mathrm{E}+02$ & $2.98 \mathrm{E}-15$ & $1.83 \mathrm{E}-12$ & $3.11 \mathrm{E}-42$ \\
\hline$f_{3}$ & $1.23 \mathrm{E}-01$ & $1.96 \mathrm{E}-05$ & $1.24 \mathrm{E}-05$ & $1.10 \mathrm{E}+05$ & $1.16 \mathrm{E}+05$ & $2.60 \mathrm{E}-28$ & $1.01 \mathrm{E}+01$ & 4.93E-34 \\
\hline$f_{4}$ & $1.01 \mathrm{E}-01$ & 8.69E-04 & $8.98 \mathrm{E}-04$ & $7.59 \mathrm{E}+01$ & $8.18 \mathrm{E}+01$ & $3.43 \mathrm{E}-15$ & $1.30 \mathrm{E}-07$ & 7.39E-35 \\
\hline$f_{5}$ & $8.42 \mathrm{E}+01$ & $2.38 \mathrm{E}+01$ & $2.06 \mathrm{E}+01$ & $2.49 \mathrm{E}+08$ & $2.16 \mathrm{E}+08$ & $2.39 \mathrm{E}+01$ & $2.81 \mathrm{E}+01$ & $2.80 \mathrm{E}+01$ \\
\hline$f_{6}$ & $5.30 \mathrm{E}+03$ & $0.00 \mathrm{E}+00$ & $0.00 E+00$ & $6.18 \mathrm{E}+04$ & $5.48 \mathrm{E}+04$ & $0.00 E+00$ & $0.00 \mathrm{E}+00$ & $0.00 \mathrm{E}+00$ \\
\hline$f_{7}$ & $6.74 \mathrm{E}-02$ & $8.80 \mathrm{E}-02$ & $9.03 \mathrm{E}-02$ & $3.24 \mathrm{E}-01$ & $4.43 \mathrm{E}+01$ & 2.91E-02 & $2.76 \mathrm{E}-03$ & 4.45E-01 \\
\hline$f_{8}$ & $8.14 \mathrm{E}+03$ & $6.09 \mathrm{E}+03$ & $4.36 \mathrm{E}+03$ & $1.06 \mathrm{E}+04$ & $1.07 \mathrm{E}+04$ & $6.86 \mathrm{E}+03$ & $6.42 \mathrm{E}+03$ & $3.56 \mathrm{E}+03$ \\
\hline$f_{9}$ & $4.49 \mathrm{E}+01$ & $3.65 \mathrm{E}+01$ & $5.27 \mathrm{E}+01$ & $3.61 \mathrm{E}+02$ & $3.12 \mathrm{E}+02$ & $2.09 \mathrm{E}+01$ & $1.21 \mathrm{E}+01$ & $1.33 \mathrm{E}+00$ \\
\hline$f_{10}$ & $1.25 \mathrm{E}+01$ & 4.49E-04 & $4.02 \mathrm{E}-04$ & $2.05 \mathrm{E}+01$ & $2.03 \mathrm{E}+01$ & $3.02 \mathrm{E}-14$ & $2.55 \mathrm{E}-14$ & $2.76 \mathrm{E}-14$ \\
\hline$f_{11}$ & 2.94E-02 & $2.47 \mathrm{E}-03$ & 7.91E-06 & $6.09 \mathrm{E}+02$ & $5.47 \mathrm{E}+02$ & $0.00 \mathrm{E}+00$ & $3.33 \mathrm{E}-16$ & $2.59 \mathrm{E}-17$ \\
\hline$f_{12}$ & $1.25 \mathrm{E}+01$ & $1.02 \mathrm{E}-08$ & $8.28 \mathrm{E}-09$ & $6.18 \mathrm{E}+08$ & $3.99 \mathrm{E}+08$ & $1.36 \mathrm{E}-31$ & $1.23 \mathrm{E}-16$ & $1.64 \mathrm{E}-32$ \\
\hline$f_{13}$ & $5.28 \mathrm{E}+01$ & $1.49 \mathrm{E}-07$ & $1.69 \mathrm{E}-07$ & $9.13 \mathrm{E}+08$ & $8.12 \mathrm{E}+08$ & $2.13 \mathrm{E}-30$ & 4.64E-16 & 3.13E-32 \\
\hline$W / t / l$ & $12 / 0 / 1$ & $10 / 1 / 2$ & $10 / 1 / 2$ & $12 / 0 / 1$ & $13 / 0 / 0$ & $9 / 1 / 3$ & $10 / 1 / 2$ & - \\
\hline
\end{tabular}
similar performance in terms of Friedman test. Note that IFA-EO achieves better performance than NaFA in terms of $f_{\mathrm{m}}$.

Table 4: Comparative performances for test functions $f_{1}-f_{13}$ with $D=30$. 
Table 5: Friedman and Quade tests of Exp. 1

\begin{tabular}{ccc}
\hline Algorithm & Friedman ranking & Quade ranking \\
\hline IFA-EO & $\mathbf{2 . 4 6 1 5}$ & $\mathbf{2 . 3 6 8 1}$ \\
ApFA [11] & 2.8462 & 3.1868 \\
NaFA [20] & $\mathbf{2 . 4 6 1 5}$ & 2.5549 \\
VSSFA [24] & 7.3077 & 7.2418 \\
WSSFA [25] & 7.6154 & 7.7363 \\
CFA [26] & 3.7308 & 3.3901 \\
MFA [27] & 3.9615 & 3.7527 \\
FA [10] & 5.6154 & 5.7692 \\
Statistic & 66.647436 & 19.524242 \\
p-value & 0 & 0 \\
\hline
\end{tabular}

Remark 2: Although the value 1.10E-108 and 1.64E-32 in Table 4 is very close, any improved performance may mean the improvements of exploration ability and exploitation ability. For example, Fig. 6 gives an example. The term "A" means the global optimum and the term "B" means the local optimum. In some real applications, it is difficult to know how many local optimums in the optimization function. If the algorithm can find "A" rather than "B", the algorithm may have good exploration ability. Similarly, the term "C" is close to global optimum, but is fails to find the global optimum "A". If the algorithm can find "A" rather than " $\mathrm{C}$ ", the algorithm may have good exploitation ability. Due to lack of knowledge of real-world optimization function, we cannot ensure every " $B$ " or "C" is very close to "A". Thus, we report these errors. For some real-world cases, below a threshold (e.g.,1.0E-8) the error can be considered as zero. In this paper, we test the exploration ability and exploitation ability of IFA-EO, thus the errors below 1.0E-8 are not considered as zero.

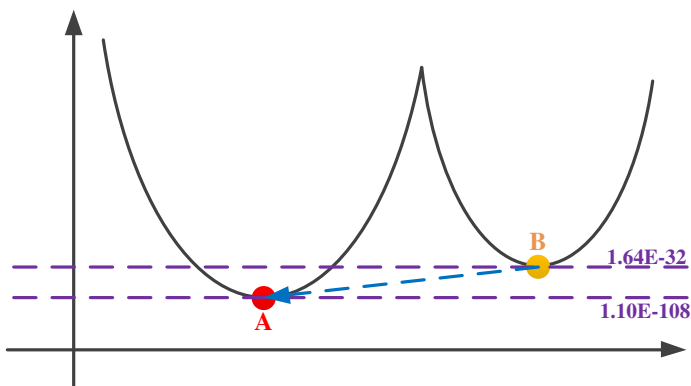

(a)

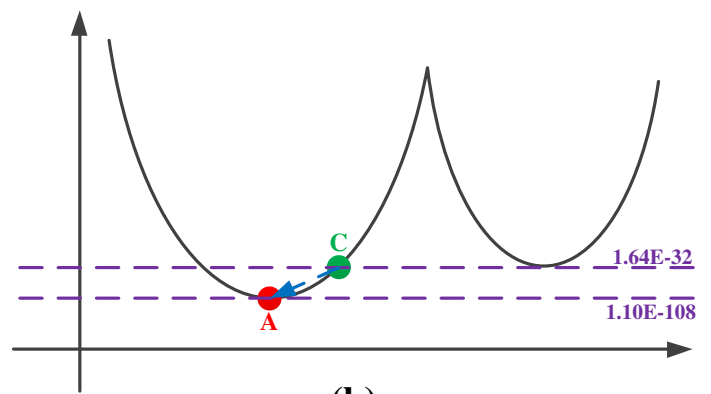

(b)

Fig. 6: Two cases of small error.

\subsection{Exp. 2: Comparison with EO-based algorithms}

This group of well-known benchmark functions shown in Table 6 is selected from the literature [20]. In this experiment, we choose three recently published related EO variants i.e., PEO [38], PSO-EO [34], and RPEO-PLM [39] as comparisons to demonstrate the effectiveness of combination of EO mechanism in FA. The experimental results of four algorithms, i.e., IFA-EO, PEO, PSO-EO, and RPEO-PLM for eight test functions are presented in Table 7. $f_{\mathrm{m}}$ represents the average results of run 30 times. The results of RPEO-PLM are taken from the reference [39]. Table 7 implies that the proposed IFA-EO algorithm performs the best in 4 out of 8 test functions $h_{1}-h_{8}$, the same as PSO-EO for functions $h_{2}$ and $h_{7}$, and the same as RPEO-PLM for function $h_{7}$. Therefore, IFA-EO algorithm has a more powerful ability to solve such high-dimension test functions $h_{1}-h_{8}$ than these three compared algorithms.

To further compare four EO-based swarm intelligence algorithms, we use KEEL [50] software to conduct the Friedman test and Quade test and the Bonfreeoni-Dunn method is selected as the post-hoc test. Table 8 gives the rankings obtained by IFA-EO and other EO-based algorithms. Based on the Friedman rankings, IFA-EO achieves the best performance. We can see that IFA-EO is obviously better than PEO and is significantly better than RPEO-PLM. Compare IFA-EO with PSO-EO, they achieve similar results. 
Table 6: Benchmark functions $h_{1}-h_{8}$ with $D=100$

\begin{tabular}{|c|c|c|c|}
\hline Problem & Function expression & Search space & Global minimum \\
\hline$h_{1}$ & $h_{1}=\sum_{i=1}^{D}\left|\frac{\sin \left(10 x_{i} \pi\right)}{10 x_{i} \pi}\right|$ & {$[-0.5,0.5]$} & 0 \\
\hline$h_{2}$ & $h_{2}=\sum^{D}\left[x_{i}+0.5\right]^{2}$ & {$[-100,100]$} & 0 \\
\hline$h_{3}$ & $h_{3}=\sum_{i=1}^{D}\left[x_{i}^{2}-10 \cos \left(2 \pi x_{i}\right)+10\right]$ & {$[-5.12,5.12]$} & 0 \\
\hline$h_{4}$ & $h_{4}=\sum_{i=1}^{D} x_{i}^{2}$ & {$[-5.12,5.12]$} & 0 \\
\hline$h_{5}$ & $h_{5}=-20 \exp \left(-0.02 \sqrt{\frac{1}{D}} \sum_{i=1}^{D} x_{i}^{2}\right)-\exp \left(\frac{1}{D} \sum_{i=1}^{D} \cos \left(2 \pi x_{i}\right)\right)+20+e$ & {$[-30,30]$} & 0 \\
\hline$h_{6}$ & $h_{6}=418.9828 D-\sum_{i=1}^{D} x_{i} \sin \left(\sqrt{\left|x_{i}\right|}\right)$ & {$[-500,500]$} & 0 \\
\hline$h_{7}$ & $h_{7}=6 D+\sum_{i=1}^{D}\left\lfloor x_{i}\right\rfloor$ & {$[-5.12,5.12]$} & 0 \\
\hline$h_{8}$ & $h_{8}=1+\frac{1}{4000} \sum_{i=1}^{D} x_{i}^{2}-\prod_{i=1}^{D} \cos \left(\frac{x_{i}}{\sqrt{i}}\right)$ & {$[-600,600]$} & 0 \\
\hline
\end{tabular}

Table 7: Comparative results on test functions $h_{1}-h_{8}$ with $D=100$.

\begin{tabular}{|c|c|c|c|c|c|c|c|c|}
\hline \multirow{2}{*}{ Function } & \multicolumn{2}{|c|}{ IFA-EO } & \multicolumn{2}{|c|}{ PEO [38] } & \multicolumn{2}{|c|}{ RPEO-PLM [39] } & \multicolumn{2}{|c|}{ PSO-EO [34] } \\
\hline & Mean & rank & Mean & rank & Mean & rank & Mean & rank \\
\hline$h_{1}$ & $3.53 \mathrm{E}-07$ & 2 & $2.62 \mathrm{E}-03$ & 4 & $1.89 \mathrm{E}-06$ & 3 & $0.00 E+00$ & 1 \\
\hline$h_{2}$ & $0.00 \mathrm{E}+00$ & 1 & $1.62 \mathrm{E}-06$ & 3 & $1.66 \mathrm{E}-05$ & 4 & $0.00 \mathrm{E}+00$ & 1 \\
\hline$h_{3}$ & $6.85 \mathrm{E}-05$ & 3 & $1.26 \mathrm{E}+01$ & 4 & $2.76 \mathrm{E}-06$ & 2 & $0.00 E+00$ & 1 \\
\hline$h_{4}$ & $1.62 \mathrm{E}-111$ & 1 & $3.60 \mathrm{E}-10$ & 3 & $1.39 \mathrm{E}-08$ & 4 & $2.15 \mathrm{E}-11$ & 2 \\
\hline$h_{5}$ & 1.07E-13 & 1 & $2.04 \mathrm{E}+01$ & 4 & $6.98 \mathrm{E}-08$ & 2 & $5.02 \mathrm{E}-05$ & 3 \\
\hline$h_{6}$ & $8.01 \mathrm{E}+03$ & 3 & $7.52 \mathrm{E}+04$ & 4 & $1.38 E-03$ & 1 & $8.62 \mathrm{E}+00$ & 2 \\
\hline$h_{7}$ & $0.00 \mathrm{E}+00$ & 1 & $3.02 \mathrm{E}+00$ & 4 & $0.00 \mathrm{E}+00$ & 1 & $0.00 \mathrm{E}+00$ & 1 \\
\hline$h_{8}$ & $6.62 \mathrm{E}-16$ & 2 & $0.00 E+00$ & 1 & $5.01 \mathrm{E}-06$ & 3 & $9.27 \mathrm{E}-04$ & 4 \\
\hline Average rank & \multicolumn{2}{|c|}{1.75} & \multicolumn{2}{|c|}{3.375} & \multicolumn{2}{|c|}{2.5} & \multicolumn{2}{|c|}{1.875} \\
\hline Final rank & \multicolumn{2}{|l|}{1} & \multicolumn{2}{|c|}{4} & \multicolumn{2}{|l|}{3} & \multicolumn{2}{|c|}{2} \\
\hline
\end{tabular}

Table 8: Friedman and Quade tests of Exp. 2

\begin{tabular}{ccc}
\hline Algorithm & Friedman ranking & Quade ranking \\
\hline IFA-EO & $\mathbf{1 . 8 7 5}$ & 2.0972 \\
RPEO-PLM [39] & 3.4375 & 3.7083 \\
PEO [38] & 2.625 & 2.1389 \\
PSO-EO [34] & 2.0625 & $\mathbf{2 . 0 5 5 6}$ \\
Statistic & 7.0875 & 6.1719 \\
$p$-value & 0.0692 & 0.00355 \\
\hline
\end{tabular}

\subsection{Exp. 3: Comparison with L-SHADE and AD-IFA}

In Exp. 3, the performance of the IFA-EO is compared with recently improved FA, called an adaptive logarithmic spiral-levy FA (AD-IFA) and advanced DE variant, called linear population size reduction technique of success history 
based adaptive differential evolution (L-SHADE). AD-IFA uses the logarithmic-spiral guidance the paths of fireflies and considers an adaptive switching to keep the suitable balance between exploration ability and exploitation ability. The effectiveness of AD-IFA has been illustrated by solving various test functions and real-life engineering problems. Thus, as one of the improved version of FA, the performance of IFA-EO can be further testified by comparing with this state-of-the-art FA algorithm. Furthermore, as an advanced DE variant, L-SHADE is considered as the competitor to show the performance of IFA-EO. The adjustable parameters of AD-IFA and L-SHADE are suggested by the corresponding references. The thirteen test functions are given in Table 3 with $D=50$. The $N P$ is set as 20 and the $F E S_{\max }$ is set as 300,000 as the stop criterion. Other parameters are the same as suggested in [37], [40]. Table 9 presents the comparative performances of IFA-EO with above two algorithms on test functions $f_{1}-f_{13}$ in terms of $f_{m}$ and SD. Symbol "(1)" "(5)" implies the rank of algorithms in terms of $f_{m}$ and SD achieved from 30 independent runs. In addition, Table 10 gives the Friedman and Quade rankings. From Tables 9 and Table 10, we can see the following observation:

(1) Through comparing IFA-EO with AD-IFA, it can be clearly observed that IFA-EO achieves all better performance than AD-IFA in solving $f_{1}-f_{13}$ in terms of $f_{m}$ and SD except the SD of $f_{1}$ and $f_{2}$.

(2) By comparing IFA-EO and L-SHADE, it is clear that IFA-EO wins in seven test functions. Also, L-SHADE achieves better performance than IFA-EO in other six functions. They achieve similar performance for $f_{1}-f_{13}$.

(3) IFA-EO obtains better ranks than AD-IFA and has similar performance with L-SHADE in the view of nonparametric statistics test.

L-SHADE is an improved version of DE and achieves better performance than various DE algorithms. Although the proposed IFA-EO achieves similar performance with L-SHADE, it significantly outperforms the recently improved version of FA, i.e., AD-IFA [37]. Thus, as one kind of FA variant, IFA-EO may be considered as a potential algorithm in evolutionary algorithms. From Table 9, it can be found the L-SHADE performs worse performance in $f_{4}, f_{9}, f_{10}, f_{12}$, and $f_{13}$ while IFA-EO performs well in solving these functions. Thus, the improvements in IFA-EO may further enhance the performance of L-SHADE.

Table 9: Comparative experimental results of $f_{1}-f_{13}$ obtained by IFA-EO, AD-FIA and L-SHADE

\begin{tabular}{|c|c|c|c|c|c|}
\hline Test function & Optimum & Algorithm & $f_{m}(\mathrm{rank})$ & SD (rank) & Final rank \\
\hline \multirow[t]{3}{*}{$f_{1}$} & $0(\min )$ & IFA-EO & $5.68 \mathrm{E}-09(2)$ & $1.02 \mathrm{E}-08(3)$ & 2 \\
\hline & & AD-IFA & $3.91 \mathrm{E}-08(3)$ & $9.42 \mathrm{E}-09(2)$ & 3 \\
\hline & & L-SHADE & $4.87 E-23(1)$ & $2.46 \mathrm{E}-22(1)$ & 1 \\
\hline \multirow[t]{3}{*}{$f_{2}$} & $0(\min )$ & IFA-EO & $4.58 \mathrm{E}-04(2)$ & $1.26 \mathrm{E}-03(3)$ & 2 \\
\hline & & AD-IFA & $2.00 \mathrm{E}-03(3)$ & $5.78 \mathrm{E}-04(2)$ & 3 \\
\hline & & L-SHADE & 2.74E-21(1) & $9.60 \mathrm{E}-21(1)$ & 1 \\
\hline \multirow[t]{3}{*}{$f_{3}$} & $0(\min )$ & IFA-EO & $7.77 \mathrm{E}-04(1)$ & $3.21 \mathrm{E}-03(1)$ & 1 \\
\hline & & AD-IFA & $6.22 \mathrm{E}+00(3)$ & $8.88 \mathrm{E}+00(3)$ & 3 \\
\hline & & L-SHADE & $2.29 \mathrm{E}-02(2)$ & $5.56 \mathrm{E}-02(2)$ & 2 \\
\hline \multirow[t]{3}{*}{$f_{4}$} & $0(\min )$ & IFA-EO & $7.12 \mathrm{E}-02(1)$ & 5.18E-02(1) & 1 \\
\hline & & AD-IFA & $1.43 \mathrm{E}+01(2)$ & $5.97 \mathrm{E}+00(3)$ & 2 \\
\hline & & L-SHADE & $3.18 \mathrm{E}+01(3)$ & $4.12 \mathrm{E}+00(2)$ & 3 \\
\hline \multirow[t]{3}{*}{$f_{5}$} & $0(\min )$ & IFA-EO & $9.12 \mathrm{E}+01(2)$ & $4.41 \mathrm{E}+01(2)$ & 2 \\
\hline & & AD-IFA & $1.06 \mathrm{E}+02(3)$ & $9.54 \mathrm{E}+01(3)$ & 3 \\
\hline & & L-SHADE & $8.77 E+01(1)$ & $4.21 \mathrm{E}+01(1)$ & 1 \\
\hline \multirow[t]{3}{*}{$f_{6}$} & $0(\min )$ & IFA-EO & $2.27 \mathrm{E}-08(2)$ & 5.39E-08(3) & 2 \\
\hline & & AD-IFA & $4.15 \mathrm{E}-08(3)$ & $1.26 \mathrm{E}-08(2)$ & 3 \\
\hline & & L-SHADE & 3.53E-22(1) & 1.30E-21(1) & 1 \\
\hline \multirow[t]{3}{*}{$f_{7}$} & $0(\min )$ & IFA-EO & $4.45 \mathrm{E}-01(2)$ & $2.86 \mathrm{E}-01(3)$ & 2 \\
\hline & & AD-IFA & $5.45 \mathrm{E}-01(3)$ & $1.60 \mathrm{E}-01(2)$ & 3 \\
\hline & & L-SHADE & $1.31 \mathrm{E}-01(1)$ & 1.03E-01(1) & 1 \\
\hline
\end{tabular}




\begin{tabular}{|c|c|c|c|c|c|}
\hline \multirow[t]{3}{*}{$f_{8}$} & $-20949(\min )$ & IFA-EO & $-1.64 \mathrm{E}+04(2)$ & $8.01 \mathrm{E}+02(2)$ & 2 \\
\hline & & AD-IFA & $-1.33 \mathrm{E}+04(3)$ & $1.35 \mathrm{E}+03(3)$ & 3 \\
\hline & & L-SHADE & $-2.03 E+04(1)$ & $2.86 E+02(1)$ & 1 \\
\hline \multirow[t]{3}{*}{$f_{9}$} & $0(\min )$ & IFA-EO & $4.30 \mathrm{E}-08(1)$ & 1.57E-07(1) & 1 \\
\hline & & AD-IFA & $9.02 \mathrm{E}+01(3)$ & $2.36 \mathrm{E}+01(3)$ & 3 \\
\hline & & L-SHADE & $9.62 \mathrm{E}-01(2)$ & $1.47 \mathrm{E}+00(2)$ & 2 \\
\hline \multirow[t]{3}{*}{$f_{10}$} & $0(\min )$ & IFA-EO & $1.66 \mathrm{E}-05(1)$ & $1.63 E-05(1)$ & 1 \\
\hline & & AD-IFA & 9.73E-01(2) & $1.02 \mathrm{E}+00(2)$ & 2 \\
\hline & & L-SHADE & $7.88 \mathrm{E}+00(3)$ & $1.16 \mathrm{E}+00(3)$ & 3 \\
\hline \multirow[t]{3}{*}{$f_{11}$} & $0(\min )$ & IFA-EO & $5.09 \mathrm{E}-03(1)$ & $6.75 E-03(1)$ & 1 \\
\hline & & AD-IFA & $6.89 \mathrm{E}-03(2)$ & $1.02 \mathrm{E}-02(2)$ & 2 \\
\hline & & L-SHADE & 1.04E-01(3) & $1.58 \mathrm{E}-01(3)$ & 3 \\
\hline \multirow[t]{3}{*}{$f_{12}$} & $0(\min )$ & IFA-EO & $1.64 \mathrm{E}-32(1)$ & $2.20 \mathrm{E}-33(1)$ & 1 \\
\hline & & AD-IFA & $2.77 \mathrm{E}+00(3)$ & $1.59 \mathrm{E}+00(2)$ & 3 \\
\hline & & L-SHADE & $2.20 \mathrm{E}+00(2)$ & $2.21 \mathrm{E}+00(3)$ & 2 \\
\hline \multirow[t]{3}{*}{$f_{13}$} & $0(\min )$ & IFA-EO & $9.70 \mathrm{E}-33(1)$ & 3.13E-32(1) & 1 \\
\hline & & AD-IFA & $2.50 \mathrm{E}+00(3)$ & $9.63+\mathrm{E} 00(3)$ & 3 \\
\hline & & L-SHADE & $1.39 \mathrm{E}+00(2)$ & $2.44 \mathrm{E}+00(2)$ & 2 \\
\hline
\end{tabular}

Table 10: Friedman and Quade tests of Exp. 3

\begin{tabular}{ccc}
\hline Algorithm & Friedman ranking & Quade ranking \\
\hline IFA-EO & $\mathbf{1 . 4 6 1 5}$ & $\mathbf{1 . 3 7 3 6}$ \\
AD-IFA [37] & 2.7692 & 2.7363 \\
L-SHADE [40] & 1.7692 & 1.8901 \\
Statistic & 12.15 & 6.99 \\
p-value & 0.002295 & 0.00405 \\
\hline
\end{tabular}

\subsection{Exp. 4: Effects of different strategies}

From the above first three experiments, we have shown the superiority of the proposed IFA-EO algorithm for two well-known benchmark functions. As described in Section 3, IFA-EO employs three strategies: a probability choice for combination of full attraction model and the single attraction model, and acceptance of some worse solutions through small probability in the single attraction model; an adaptive step with iterations; and the combination of EO procedure. To assess the performance of these three strategies, we compare five FAs equipped with different strategies in the fourth experiment to verify the influence on the performance of the IFA-EO algorithm. The considered algorithms are given as follows.

- $\mathrm{FA}+$ Beta (termed as FA-B)

- $\mathrm{FA}+$ Beta + adaptive step (termed as FA-A)

- $\mathrm{FA}+\mathrm{Beta}+\mathrm{EO}$ procedure (termed as FA-EO)

- $\mathrm{FA}+$ Beta + probability choice + adaptive step (termed as FA-PA)

- $\mathrm{FA}+\mathrm{Beta}+$ probability choice + adaptive step + EO procedure (i.e., IFA-EO)

In this experiment, all algorithms use the same parameter settings as described in Sec. 4.1. And INV set as 5 in the FA-EO for all test functions.

Table 11: Comparative results for test functions $f_{1-}-f_{13}$ with $D=30$

\begin{tabular}{cccccc}
\hline \multicolumn{2}{c}{ Function } & IFA-EO & FA-PA & FA-A & FA-EO \\
\hline & $f_{b}$ & $3.81 \mathrm{E}-14$ & $\mathbf{1 . 9 5 E - 1 4}$ & $2.97 \mathrm{E}-04$ & $2.75 \mathrm{E}-02$ \\
\multirow{2}{*}{$f_{1}$} & $f_{w}$ & $\mathbf{4 . 1 6 E - 1 0}$ & $1.97 \mathrm{E}-08$ & $5.12 \mathrm{E}-04$ & $5.38 \mathrm{E}-02$ \\
& $f_{m}$ & $\mathbf{5 . 6 6 E - 1 1}$ & $7.49 \mathrm{E}-10$ & $5.10 \mathrm{E}-04$ & $3.74 \mathrm{E}-02$
\end{tabular}




\begin{tabular}{|c|c|c|c|c|c|c|}
\hline \multirow{3}{*}{$f_{2}$} & $f_{b}$ & $8.81 \mathrm{E}-06$ & $5.96 \mathrm{E}-06$ & 7.66E-03 & 4.66E-01 & $9.27 \mathrm{E}-01$ \\
\hline & $f_{w}$ & $1.07 \mathrm{E}-03$ & $3.48 \mathrm{E}-03$ & $1.46 \mathrm{E}-02$ & $8.47 \mathrm{E}-01$ & $1.08 \mathrm{E}+0 \mathrm{C}$ \\
\hline & $f_{m}$ & $2.40 \mathrm{E}-04$ & $3.90 \mathrm{E}-04$ & $1.15 \mathrm{E}-02$ & $6.57 \mathrm{E}-01$ & $1.01 \mathrm{E}+0 \mathrm{C}$ \\
\hline \multirow{3}{*}{$f_{3}$} & $f_{b}$ & $1.24 \mathrm{E}+01$ & $1.86 \mathrm{E}+01$ & $2.68 \mathrm{E}+00$ & $1.83 \mathrm{E}-01$ & $1.54 \mathrm{E}-01$ \\
\hline & $f_{w}$ & $2.10 \mathrm{E}+02$ & $3.35 \mathrm{E}+02$ & $9.62 \mathrm{E}+01$ & $1.49 \mathrm{E}+00$ & $2.05 \mathrm{E}+0 \mathrm{C}$ \\
\hline & $f_{m}$ & $8.02 \mathrm{E}+01$ & $1.14 \mathrm{E}+02$ & $2.51 \mathrm{E}+01$ & $6.36 \mathrm{E}-01$ & $5.35 \mathrm{E}-01$ \\
\hline \multirow{3}{*}{$f_{4}$} & $f_{b}$ & $2.27 \mathrm{E}-04$ & 4.66E-04 & $1.89 \mathrm{E}-02$ & $1.30 \mathrm{E}+00$ & $6.66 \mathrm{E}-01$ \\
\hline & $f_{w}$ & $3.69 \mathrm{E}-03$ & $2.58 \mathrm{E}-03$ & $3.70 \mathrm{E}-02$ & $1.01 \mathrm{E}+01$ & $1.03 \mathrm{E}+01$ \\
\hline & $f_{m}$ & $1.18 E-03$ & $1.23 \mathrm{E}-03$ & $2.90 \mathrm{E}-02$ & $5.00 \mathrm{E}+00$ & $5.34 \mathrm{E}+00$ \\
\hline \multirow{3}{*}{$f_{5}$} & $f_{b}$ & $2.58 \mathrm{E}+01$ & $2.53 \mathrm{E}+01$ & $2.52 \mathrm{E}+01$ & $2.81 \mathrm{E}+01$ & $2.95 \mathrm{E}+01$ \\
\hline & $f_{w}$ & $1.38 \mathrm{E}+02$ & $1.24 \mathrm{E}+03$ & $4.21 \mathrm{E}+02$ & $1.66 \mathrm{E}+02$ & $1.45 \mathrm{E}+02$ \\
\hline & $f_{m}$ & $4.99 \mathrm{E}+01$ & $1.36 \mathrm{E}+02$ & $6.61 \mathrm{E}+01$ & $4.36 \mathrm{E}+01$ & $3.97 \mathrm{E}+01$ \\
\hline \multirow{3}{*}{$f_{6}$} & $f_{b}$ & $0.00 \mathrm{E}+00$ & $0.00 \mathrm{E}+00$ & $0.00 \mathrm{E}+00$ & $0.00 \mathrm{E}+00$ & $2.23 \mathrm{E}+03$ \\
\hline & $f_{w}$ & $1.00 \mathrm{E}+00$ & $2.00 \mathrm{E}+00$ & $1.00 \mathrm{E}+00$ & $2.30 \mathrm{E}+01$ & $8.02 \mathrm{E}+03$ \\
\hline & $f_{m}$ & $6.67 \mathrm{E}-02$ & $1.67 \mathrm{E}-01$ & $1.67 \mathrm{E}-01$ & $5.60 \mathrm{E}+00$ & $4.82 \mathrm{E}+03$ \\
\hline \multirow{3}{*}{$f_{7}$} & $f_{b}$ & $3.06 \mathrm{E}-03$ & $9.64 \mathrm{E}-02$ & 3.43E-02 & $1.77 \mathrm{E}-02$ & $1.74 \mathrm{E}-01$ \\
\hline & $f_{w}$ & $9.93 \mathrm{E}-01$ & $9.78 \mathrm{E}-01$ & $9.56 \mathrm{E}-01$ & $9.75 \mathrm{E}-01$ & $9.54 \mathrm{E}-01$ \\
\hline & $f_{m}$ & 4.82E-01 & $5.31 \mathrm{E}-01$ & $4.89 \mathrm{E}-01$ & 4.31E-01 & $5.64 \mathrm{E}-01$ \\
\hline \multirow{3}{*}{$f_{8}$} & $f_{b}$ & $3.07 \mathrm{E}+03$ & $4.26 \mathrm{E}+03$ & $3.14 \mathrm{E}+03$ & $5.84 \mathrm{E}+03$ & $5.23 \mathrm{E}+03$ \\
\hline & $f_{w}$ & $6.59 \mathrm{E}+03$ & $6.61 \mathrm{E}+03$ & $6.99 \mathrm{E}+03$ & $9.64 \mathrm{E}+03$ & $1.02 \mathrm{E}+04$ \\
\hline & $f_{m}$ & $5.02 \mathrm{E}+03$ & $5.33 \mathrm{E}+03$ & $5.06 \mathrm{E}+03$ & $7.79 \mathrm{E}+03$ & $7.92 \mathrm{E}+03$ \\
\hline \multirow{3}{*}{$f_{9}$} & $f_{b}$ & $1.20 \mathrm{E}+01$ & $1.59 \mathrm{E}+01$ & $1.79 \mathrm{E}+01$ & $8.80 \mathrm{E}+00$ & $2.25 \mathrm{E}+01$ \\
\hline & $f_{w}$ & $5.37 \mathrm{E}+01$ & $5.27 \mathrm{E}+01$ & $4.38 \mathrm{E}+01$ & $2.64 \mathrm{E}+01$ & $4.67 \mathrm{E}+01$ \\
\hline & $f_{m}$ & $2.86 \mathrm{E}+01$ & $2.91 \mathrm{E}+01$ & $2.95 \mathrm{E}+01$ & $1.71 \mathrm{E}+01$ & $3.39 \mathrm{E}+01$ \\
\hline \multirow{3}{*}{$f_{10}$} & $f_{b}$ & $1.42 \mathrm{E}-07$ & $4.20 \mathrm{E}-08$ & $3.99 \mathrm{E}-03$ & $5.00 \mathrm{E}+00$ & $9.20 \mathrm{E}+00$ \\
\hline & $f_{w}$ & $1.90 \mathrm{E}-05$ & $4.17 \mathrm{E}-05$ & $6.17 \mathrm{E}-03$ & $8.59 \mathrm{E}+00$ & $1.45 \mathrm{E}+01$ \\
\hline & $f_{m}$ & $2.00 \mathrm{E}-06$ & $2.63 \mathrm{E}-06$ & $4.96 \mathrm{E}-03$ & $6.72 \mathrm{E}+00$ & $1.14 \mathrm{E}+01$ \\
\hline \multirow{3}{*}{$f_{11}$} & $f_{b}$ & $4.37 \mathrm{E}-10$ & $4.54 \mathrm{E}-10$ & $1.21 \mathrm{E}-03$ & $3.54 \mathrm{E}+00$ & $4.75 \mathrm{E}+00$ \\
\hline & $f_{w}$ & 3.23E-06 & $1.12 E-06$ & $1.18 \mathrm{E}-02$ & $2.49 \mathrm{E}+01$ & $2.98 \mathrm{E}+01$ \\
\hline & $f_{m}$ & 2.41E-07 & 2.13E-07 & $2.58 \mathrm{E}-03$ & $1.48 \mathrm{E}+01$ & $1.55 \mathrm{E}+01$ \\
\hline \multirow{3}{*}{$f_{12}$} & $f_{b}$ & $1.66 \mathrm{E}-12$ & $8.30 \mathrm{E}-12$ & $6.12 \mathrm{E}-06$ & $2.00 \mathrm{E}+00$ & $1.49 \mathrm{E}+00$ \\
\hline & $f_{w}$ & $2.36 \mathrm{E}-09$ & $2.94 \mathrm{E}-09$ & $2.12 \mathrm{E}-05$ & $5.44 \mathrm{E}+00$ & $1.78 \mathrm{E}+01$ \\
\hline & $f_{m}$ & $5.93 \mathrm{E}-10$ & 7.64E-10 & $1.33 \mathrm{E}-05$ & $4.24 \mathrm{E}+00$ & $9.33 \mathrm{E}+00$ \\
\hline \multirow{3}{*}{$f_{13}$} & $f_{b}$ & $4.30 \mathrm{E}-12$ & $2.98 \mathrm{E}-12$ & $6.19 \mathrm{E}-05$ & $8.81 \mathrm{E}+00$ & $3.97 \mathrm{E}+01$ \\
\hline & $f_{w}$ & $1.57 \mathrm{E}-08$ & $1.75 \mathrm{E}-08$ & $1.85 \mathrm{E}-04$ & $3.53 \mathrm{E}+01$ & $7.68 \mathrm{E}+01$ \\
\hline & $f_{m}$ & $3.64 \mathrm{E}-09$ & 4.30E-09 & $1.05 \mathrm{E}-04$ & $1.82 \mathrm{E}+01$ & $5.31 \mathrm{E}+01$ \\
\hline
\end{tabular}

Table 11 gives the competitive results of compared five algorithms. Table 12 lists the mean ranks and the results of Friedman and Quade tests, respectively. In addition, Figs.7-12 show the convergence curves of FA-B, FA-A, FA-EO, FA-PA, and IFA-EO on the selected functions. From Tables 11-12 and Figs.7-12, we can see the following observations:

(a) Through comparing IFA-EO with FA-PA, it can be clearly observed that combination of EO procedure can improve the accuracy of FA-B.

(b) By comparing FA-B with FA-A, FA-PA and IFA-EO, it can be found that the three considered strategies play a vital role in improving the performance of FA-B. The adaptive step strategy can significantly improve FA's performance, combination with probability choice and adaptive step strategy or hybrid three strategies can better enhance the ability 
of FA-B.

(c) From Table 11 and Table 12, IFA-EO achieves the best rank in terms of Friedman test and Quade test and is significantly better than other four compared algorithms.

(d) From Figs. 7-12, we can see that the accuracy of IFA-EO and FA-PA methods is higher than other different strategies. And IFA-EO is better than FA-PA method on the whole. In addition, the convergences of IFA-EO and FA-PA are faster than other compared methods.

Overall, each strategy has different impacts and plays a vital role in the algorithm, and IFA-EO can be considered the best method among the all different strategies in terms of Friedman and Quade tests, convergence speed, and global minimum value.

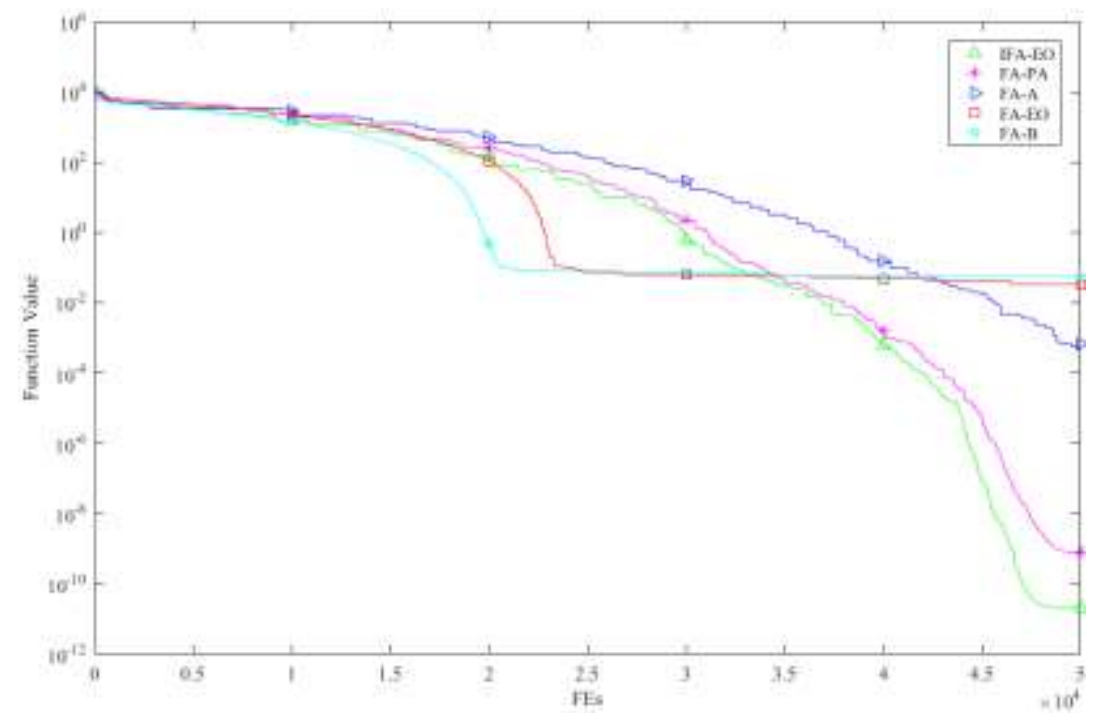

Fig. 7. Convergence curves of different FAs for $f_{1}$ test function.

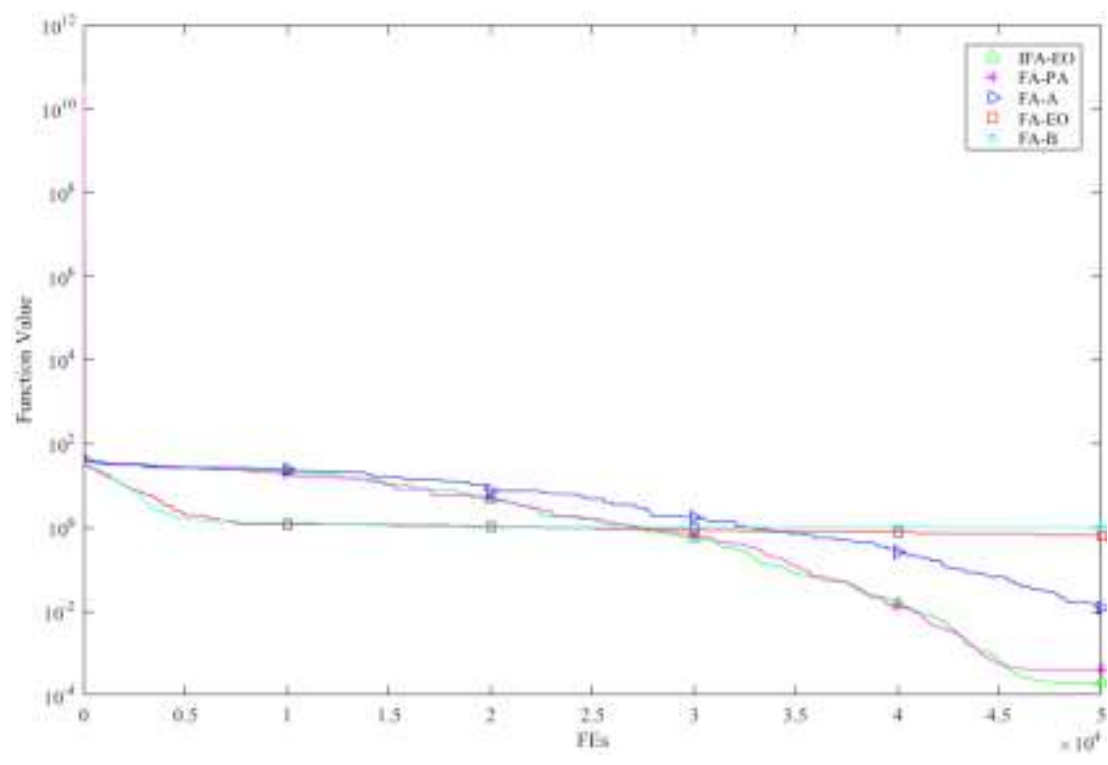

Fig. 8. Convergence curves of different FAs for $f_{2}$ test function. 


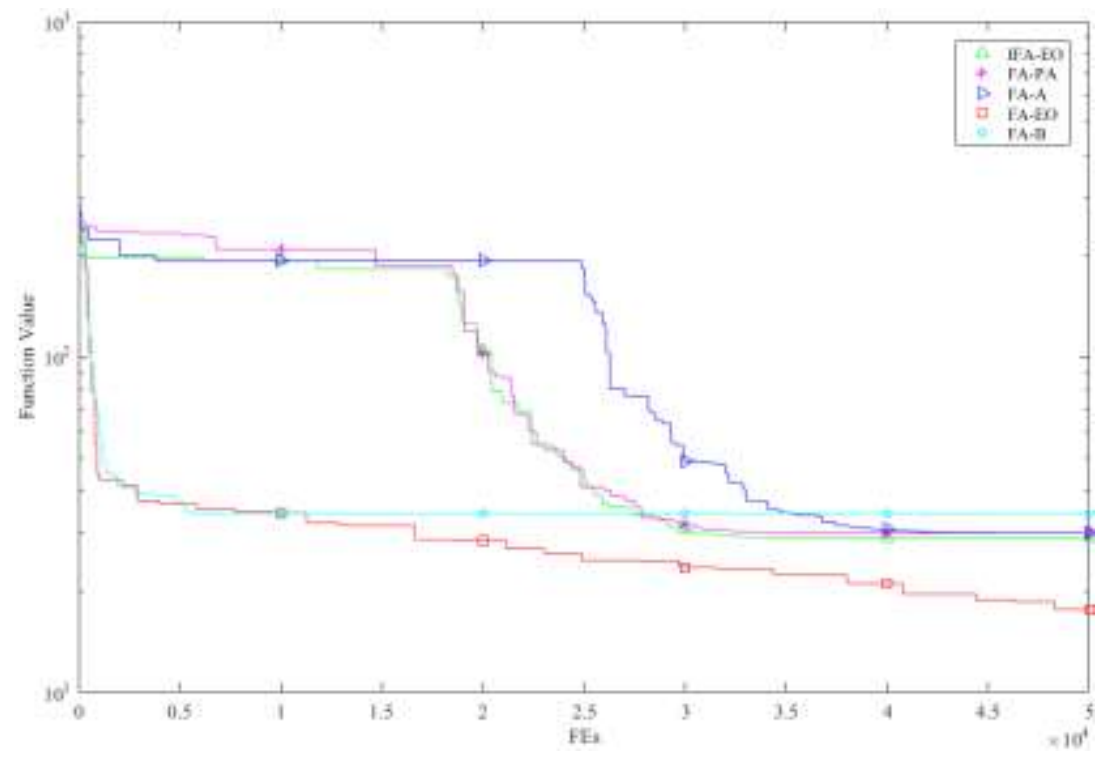

Fig. 9. Convergence curves of different FAs for $f_{9}$ test function.

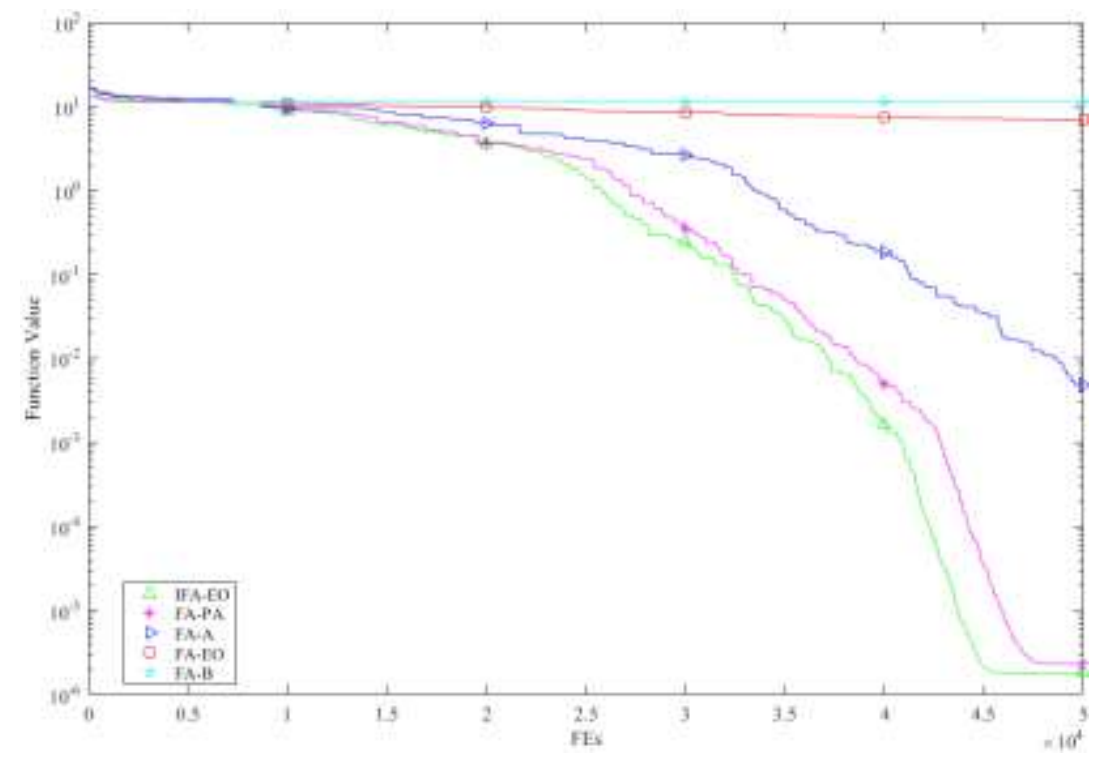

Fig. 10. Convergence curves of different FAs for $f_{10}$ test function.

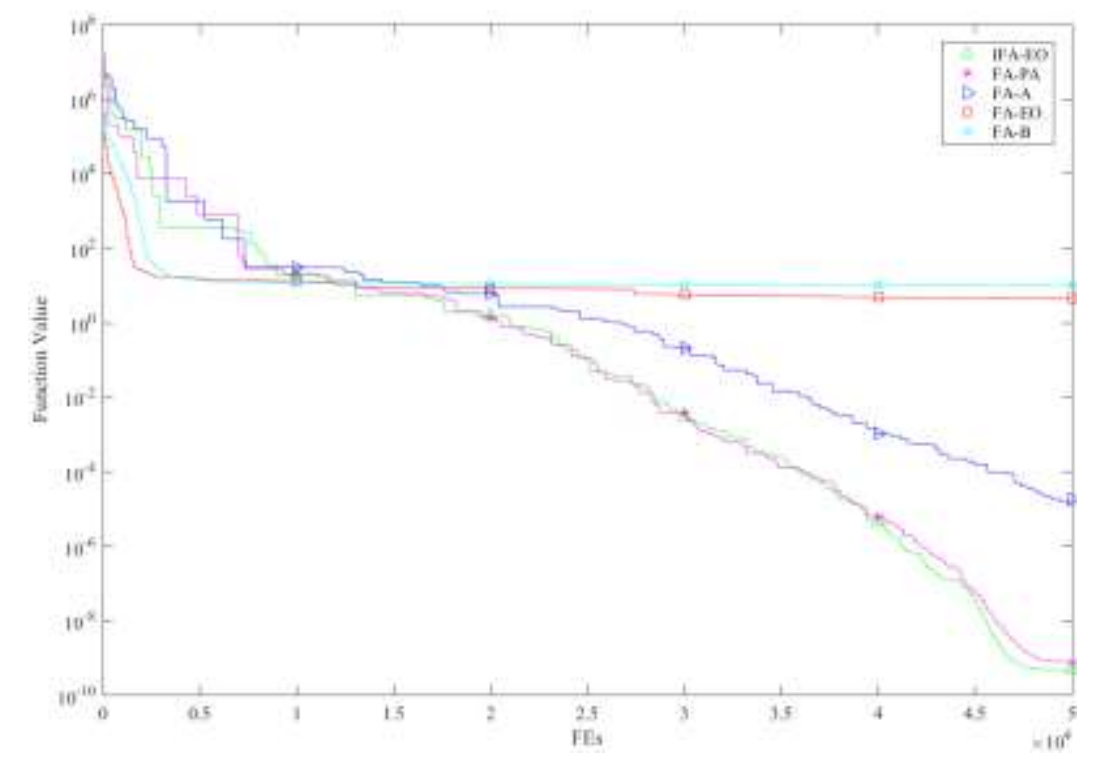

Fig. 11. Convergence curves of different FAs for $f_{12}$ test function. 


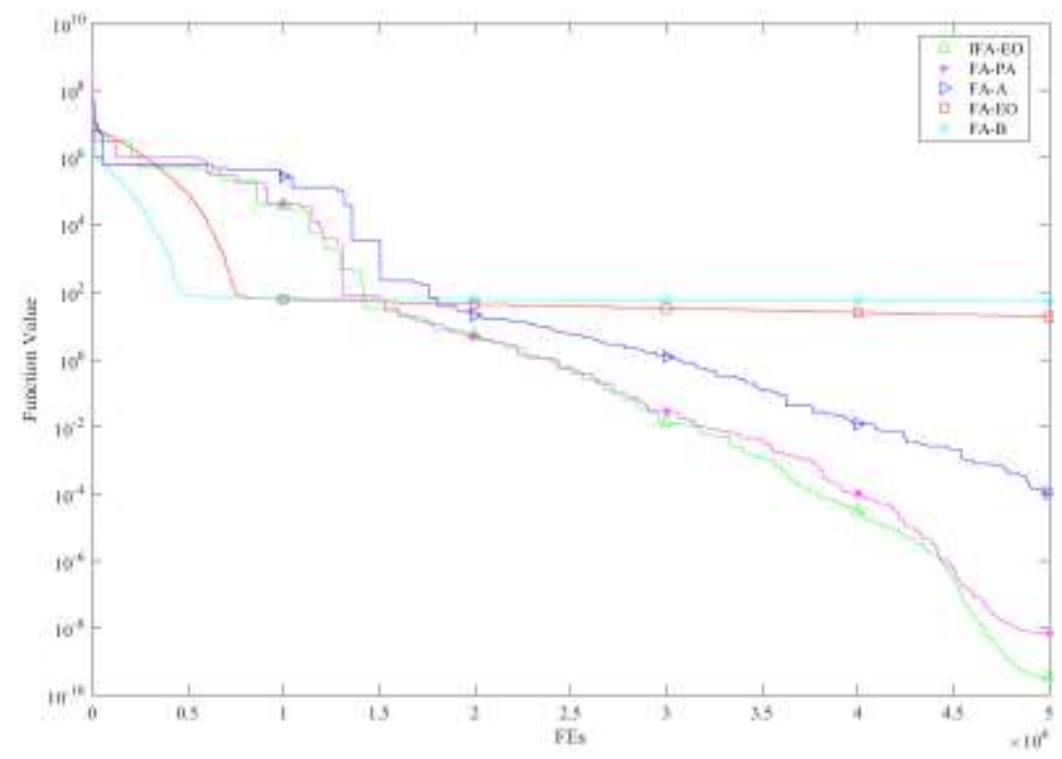

Fig. 12. Convergence curves of different FAs for $f_{13}$ test function.

Table 12: Friedman and Quade tests of Exp. 4

\begin{tabular}{ccc}
\hline Algorithm & Friedman ranking & Quade ranking \\
\hline IFA-EO & $\mathbf{1 . 6 1 5 4}$ & $\mathbf{1 . 7 6 9 2}$ \\
FA-PA & 2.7308 & 2.9505 \\
FA-A & 3.0385 & 2.9945 \\
FA-EO & 3.2308 & 3.2088 \\
FA-B & 4.3846 & 4.0769 \\
Statistic & 20.6 & 3.2207 \\
$p$-value & 0.00038 & 0.02021 \\
\hline
\end{tabular}

\subsection{Exp. 5: Effects of population size}

\begin{tabular}{|c|c|c|c|c|}
\hline \multirow{2}{*}{ Function } & \multicolumn{4}{|c|}{ IFA-EO } \\
\hline & 10 & 20 & 30 & 40 \\
\hline$f_{1}$ & $1.38 \mathrm{E}-96$ & $1.10 E-108$ & $2.77 \mathrm{E}-15$ & $6.42 \mathrm{E}-02$ \\
\hline$f_{2}$ & 6.67E-06 & 3.11E-42 & $1.97 \mathrm{E}-08$ & $1.31 \mathrm{E}-01$ \\
\hline$f_{3}$ & $3.45 \mathrm{E}-01$ & 4.93E-34 & $6.13 \mathrm{E}-05$ & $3.88 \mathrm{E}+01$ \\
\hline$f_{4}$ & 1.49E-05 & 7.39E-35 & $2.52 \mathrm{E}-07$ & $1.32 \mathrm{E}-01$ \\
\hline$f_{5}$ & $3.75 \mathrm{E}+01$ & $2.80 \mathrm{E}+01$ & $3.69 \mathrm{E}+01$ & $3.39 \mathrm{E}+01$ \\
\hline$f_{6}$ & $0.00 \mathrm{E}+00$ & $0.00 \mathrm{E}+00$ & $0.00 \mathrm{E}+00$ & $0.00 \mathrm{E}+00$ \\
\hline$f_{7}$ & 5.57E-01 & 4.45E-01 & $5.69 \mathrm{E}-01$ & $5.67 \mathrm{E}-01$ \\
\hline$f_{8}$ & $2.17 E+03$ & $3.56 \mathrm{E}+03$ & $3.47 \mathrm{E}+03$ & $3.53 \mathrm{E}+03$ \\
\hline$f_{9}$ & 4.74E-02 & $1.33 \mathrm{E}+00$ & $2.12 \mathrm{E}+01$ & $1.61 \mathrm{E}+01$ \\
\hline$f_{10}$ & $4.80 \mathrm{E}-14$ & $2.76 \mathrm{E}-14$ & 5.30E-09 & $8.84 \mathrm{E}-02$ \\
\hline$f_{11}$ & $1.40 \mathrm{E}-03$ & 2.59E-17 & $1.10 \mathrm{E}-13$ & $1.75 \mathrm{E}-01$ \\
\hline$f_{12}$ & $3.63 \mathrm{E}-06$ & $1.64 E-32$ & $1.35 \mathrm{E}-15$ & $3.22 \mathrm{E}-04$ \\
\hline$f_{13}$ & $2.42 \mathrm{E}-06$ & 3.13E-32 & $1.48 \mathrm{E}-14$ & $4.35 \mathrm{E}-03$ \\
\hline
\end{tabular}


This experiment is devoted to investigating the impacts of population size on the performance of our algorithm IFA-EO. The population size is set to $10,20,30$, and 40, respectively, while other adjustable parameters are the same as those in previous experiments.

Table 13 gives the mean best fitness values obtained by IFA-EO under different population sizes. It can see that the population size has a large impact on the precision of the final solutions on test functions. When the population size is set 20, IFA-EO can achieve better comprehensive solutions than other population size settings. Thus, in FA, we usually set the population size as 20 .

\section{Conclusions and future work}

In this paper, we present an improved firefly algorithm, namely IFA-EO, which uses three improved strategies. First of all, we use the probability choice strategy to select the attractive model of firefly, which can be divided into two models: full attraction and single attraction. Full attraction needs to be compared with all fireflies, which indicates the convergence speed is slow and it takes more time. The single attraction mode is fast, but easy to fall into local optimum. Therefore, these two models are combined by the probability formula to achieve complementarity, and in the single attraction mode, the small probability accepts the worse solution. This method can dynamically balance exploration and exploitation, which speeds up convergence and saves time. Second, instead of using a fixed step size, this paper uses an adaptive step size related to the number of iterations. The value of the previous step is relatively large and is almost zero near the end. Third, EO algorithm is considered in this paper to enhance the local search ability, and solution accuracy. When the optimal solution is unchanged continuously for $I N V$-iterations remain unchanged, EO algorithm is introduced. In the experimental part, we use two group well-known test functions to compare IFA-EO with the other thirteen algorithms. It can be seen that the IFA-EO algorithm has better performance in terms of convergence speed, solution accuracy and statistical tests. In future research work, we will try to apply the modified algorithm to some constrained continuous optimization problems and multi-objective combination optimization problems, binary optimization problems, and fuzzy clustering problems [51], [52].

\section{Acknowledgments}

This work was supported by National Natural Science Foundation of China (Nos. 61872153 and 61972288), Natural Science Foundation of Guangdong Province (No.2018A030313318), and Key-Area Research and Development Program of Guangdong Province (No. 2020B0101090004).

\section{Compliance with ethical standards}

Conflict of Interest: Min-Rong Chen declares that she has no conflict of interest. Liu-Qing Yang declares that she has no conflict of interest. Guo-Qiang Zeng declares that he has no conflict of interest. Kang-Di Lu declares that he has no conflict of interest. Yi-Yuan Huang declares that she has no conflict of interest.

Ethical approval: This article does not contain any studies with human participants or animals performed by any of the authors.

Informed consent: Informed consent was obtained from all individual participants included in the study.

\section{References}

[1] G. Beni and J. Wang, "Swarm intelligence in cellular robotic systems," Robots and biological systems: towards a new bionics?. Springer, Berlin, Heidelberg, pp.703-712, 1993.

[2] E. Bonabeau, M. Dorigo, and G. Theraulaz, "Swarm intelligence: from natural to artificial systems," NY: Oxford university press, NewYork 1999.

[3] J. Kennedy, "Particle swarm optimization," Encyclopedia of machine learning, pp. 760-766, 2010.

[4] K. Deb, A. Pratap, and S. Agarwal, "A fast and elitist multiobjective genetic algorithm: NSGA-II," IEEE Transactions on Evolutionary Computation, vol. 6 no. 2, pp.182-197, 2002.

[5] F. B. Ozsoydan, İ. Gölcük, "Cuckoo Search Algorithm with Various Walks,"Applications of Cuckoo Search Algorithm and its Variants. Springer, Singapore, pp. 47-77, 2021.

[6] X.S. Yang, "A new metaheuristic bat-inspired algorithm," Nature inspired cooperative strategies for optimization, Springer, Berlin, Heidelberg, pp. 65-74, 2010. 
[7] F. B. Ozsoydan, "Effects of dominant wolves in grey wolf optimization algorithm," Applied Soft Computing, vol. 83, 105658, 2019.

[8] İ. Gölcük, F. B. Ozsoydan, "Evolutionary and adaptive inheritance enhanced Grey Wolf Optimization algorithm for binary domains," Knowledge-Based Systems, vol. 194, 105586, 2020.

[9] F. B. Ozsoydan and A. Baykasoglu, "Analysing the effects of various switching probability characteristics in flower pollination algorithm for solving unconstrained function minimization problems," Neural Computing and Applications, vol. 31, no. 11, pp. 7805-7819, 2019.

[10] X. S. Yang, "Nature-inspired metaheuristic algorithms," Luniver press, 2010.

[11] H. Wang, X. Zhou, H. Sun, X. Yu, J. Zhao, and L. Cui, "Firefly algorithm with adaptive control parameters,” Soft Computing, vol. 21 no. 17 pp. $5091-5102,2017$.

[12] F. B. Ozsoydan and A. Baykasoglu, "A multi-population firefly algorithm for dynamic optimization problems.” IEEE International Conference on Evolving and Adaptive Intelligent Systems (EAIS). IEEE, 1-7, 2015.

[13] H. Wang, W. Wang, Z. Cui, X. Zhou, J. Zhao, and Y. Li, "A new dynamic firefly algorithm for demand estimation of water resources," Information Sciences, vol. 438, pp. 95-106, 2018.

[14] B. K. Patle, A. Pandey, A. Jagadeesh, and D.R. Parhi, "Path planning in uncertain environment by using firefly algorithm," Defence technology vol. 14, no.6, pp. 691-701, 2018.

[15] M. Alweshah and S. Abdullah, "Hybridizing firefly algorithms with a probabilistic neural network for solving classification problems," Applied Soft Computing, vol. 35, pp. 513-524, 2015.

[16] M. H. Horng, "Vector quantization using the firefly algorithm for image compression," Expert Systems with Applications, vol. 39, no. 1, pp. 1078-1091, 2012.

[17] A. Kazem, E. Sharifi, F.K. Hussain, M. Saberi, and O.K. Hussain, "Support vector regression with chaos-based firefly algorithm for stock market price forecasting," Applied Soft Computing, vol. 13, no. 2, pp. 947-958, 2013.

[18] F. B. Ozsoydan, A. Baykasoğlu, "Quantum firefly swarms for multimodal dynamic optimization problems.” Expert Systems with Applications, vol. 115, pp. 189-199, 2019.

[19] C. Zhao, C. Wu, J. Chai, X. Wang, X. Yang, J. Lee, and M.J. Kim, "Decomposition-based multi-objective firefly algorithm for RFID network planning with uncertainty, ” Applied Soft Computing, vol. 55, pp.549-564, 2017.

[20] H. Wang, W. Wang, X. Zhou, H. Sun, J. Zhao, X. Yu, and Z. Cui, "Firefly algorithm with neighborhood attraction," Information Sciences, vol. 382, pp. 374-387, 2017.

[21] H. Wang, W. Wang, H. Sun and S. Rahanmayan, "Firefly algorithm with random attraction," International Journal of Bio-Inspired Computation, vol. 8, no. 1, pp. 33-41, 2016.

[22] H. Jian, X. Chen, and D. Wu, “A switch-mode firefly algorithm for global optimization,” IEEE Access, vol. 6, pp. 54177-54184, 2018.

[23] I. Fister, X.S. Yang, J. Brest and I. Fister. Jr, "Modified firefly algorithm using quaternion representation,” Expert Systems with Applications, vol. 40, no. 18, pp. 7220-7230, 2013.

[24] S.Yu, S. Zhu, Y. Ma, and D. Mao, “A variable step size firefly algorithm for numerical optimization,” Applied Mathematics and Computation, vol. 263 , pp. 214-220, 2015

[25] S. Yu, S. Su, Q. Lu, and L. Huang, “A novel wise step strategy for firefly algorithm,” International Journal of Computer Mathematics, vol. 91, no. 12 , pp. $2507-2513,2014$..

[26] A. H. Gandomi, X. S. Yang, S. Talatahari, and A.H. Alavi, "Firefly algorithm with chaos," Communications in Nonlinear Science and Numerical Simulation, vol. 18, no. 1 pp. 89-98, 2013.

[27] I. F. Jr, X. S. Yang, I. Fister, and J. Brest, “Memetic firefly algorithm for combinatorial optimization,” arXiv preprint arXiv:1204.5165, 2012.

[28] D.H. Wolpert, W.G. Macready, No free lunch theorems for optimization, IEEE Transactions Evolution Computation, vol. 1 pp. 67-82, 1997.

[29] S. Boettcher and A. G. Percus, "Extremal optimization: methods derived from co-evolution," in: Proceedings of the Genetic and Evolutionary Computation Conference, pp.825-832, 1999.

[30] S. Boettcher and A. G. Percus, "Nature’s way of optimizing," Artificial Intelligence, vol. 119, pp. 275-286, 2000.

[31] G. Q. Zeng, X. Q. Xie, M. R. Chen, and J. Weng, “Adaptive population extremal optimization-based PID neural network for multivariable nonlinear control systems," Swarm and evolutionary computation, vol. 44, pp.320-334, 2019.

[32] G. Q. Zeng, J. Chen, Y. X. Dai, L. M. Li, C. W. Zheng, and M.R. Chen, "Design of fractional order PID controller for automatic regulator voltage system based on multi-objective extremal optimization," Neurocomputing, vol. 160, pp. 173-184, 2015.

[33] K. D. Lu, W.N. Zhou, G.Q. Zeng, and Y.Y. Zheng, "Constrained population extremal optimization-based robust load frequency control of 
multi-area interconnected power system," International Journal of Electrical Power \& Energy Systems, vol. 105, pp. 249-271, 2019.

[34] M. R. Chen, X. Li, X. Zhang, and Y.Z. Lu, “A novel particle swarm optimizer hybridized with extremal optimization,” Applied Soft Computing, vol. 10 , no. 2 , pp. $367-373,2010$.

[35] M. R. Chen, J. H. Chen, G. Q. Zeng, K. D. Lu, and X. F. Jiang, "An improved artificial bee colony algorithm combined with extremal optimization and Boltzmann Selection probability". Swarm and Evolutionary Computation, vol. 49, pp. 158-177, 2019.

[36] X. Pan, L. Xue, and R. Li, “A new and efficient firefly algorithm for numerical optimization problems," Neural Computing and Applications, pp. 1-9, 2018.

[37] J. Wu, Y. G. Wang, K. Burrage, Y. C. Tian, B. Lawson, Z. Ding, “An improved firefly algorithm for global continuous optimization problems,” Expert Systems with Applications, 113340, 2020.

[38] M. R. Chen, Y. Z. Lu, and G. K. Yang, "Population-based extremal optimization with adaptive Lévy mutation for constrained optimization," in: Proceedings of 2006 International Conference on Computational Intelligence and Security (CIS'06), pp. 258-261, 2006.

[39] L. M. Li, K. D. Lu, G. Q. Zeng, L. Wu, and M. R. Chen, "A novel real-coded population-based extremal optimization algorithm with polynomial mutation: A non-parametric statistical study on continuous optimization problems,” Neurocomputing, vol. 174, pp. 577-587, 2016.

[40] P. P. Biswas, P. N. Suganthan, G. Wu, and G.A.J Amaratunga, "Parameter estimation of solar cells using datasheet information with the application of an adaptive differential evolution algorithm," Renewable Energy, vol. 132, pp. 425-438, 2019.

[41] P. H. Tang and M. H. Tseng, “Adaptive directed mutation for real-coded genetic algorithms,” Applied Soft Computing, vol. 13, pp. 600-614, 2013.

[42] S. Y. Ho, L. S. Shu, and J. H. Chen, "Intelligent evolutionary algorithms for large parameter optimization problems," IEEE Transactions on Evolutionary Computation, vol. 8, no. 6, pp. 522-541, 2004.

[43] R. Storn and K. Price, "Differential evolution-A simple and efficient heuristic for global optimization over continuous spaces," Journal of Global Optimization, vol. 11, no. 4, pp. 341-359, 1997.

[44] J. Olamaei, M. Moradi, and T. Kaboodi, "A new adaptive modified firefly algorithm to solve optimal capacitor placement problem," in: Proceedings of the 2013 18th Conference on Electrical Power Distribution Networks (EPDC), pp. 1-6, 2013.

[45] B. Bhushan and S.S. Pillai, "Particle swarm optimization and firefly algorithm: performance analysis," in: IEEE International Advance Computing Conference (IACC). IEEE, pp. 746-751, 2013.

[46] G.A. Trunfio, "Enhancing the firefly algorithm through a cooperative coevolutionary approach: an empirical study on benchmark optimisation problems, " International Journal of Bio-Inspired Computation, vol. 6, no. 2, pp. 108-125, 2014

[47] S.X. Lv, Y. R. Zeng, and L. Wang, "An effective fruit fly optimization algorithm with hybrid information exchange and its applications," International Journal of Machine Learning and Cybernetics, vol. 9, no. 10, pp. 1623-1648, 2018.

[48] T. Chen, J. He, G. Sun, G. Chen, X. Yao, “A new approach for analyzing average time complexity of population-based evolutionary algorithms on unimodal problems," IEEE Transactions on Systems, Man, and Cybernetics, Part B (Cybernetics), vol. 39, no. 5, pp.1092-1106, 2009.

[49] J. Derrac, S. García, D. Molina, and F. Herrers, "A practical tutorial on the use of nonparametric statistical tests as a methodology for comparing evolutionary and swarm intelligence algorithms," Swarm and Evolutionary Computation, vol. 1, no. 1, pp. 3-18, 2011.

[50] J. Alcalá-Fdez, L. Sánchez, S. Garcia, M.J.del. Jesus, S. Ventura, J.M. Garrell, J. Otero, C. Romero, J. Bacardit, V.M. Rivas, J.C. Fernandez, and F. Herrera., KEEL: a software tool to assess evolutionary algorithms for data mining problems, Soft Computing, vol. 13, no. 3 pp. 307-318, 2009.

[51] F. B. Ozsoydan, "Artificial search agents with cognitive intelligence for binary optimization problems," Computers \& Industrial Engineering, vol. 136, pp. 18-30, 2019.

[52] A. Baykasoğlu, İ. Gölcük, and F. B. Özsoydan, "Improving fuzzy c-means clustering via quantum-enhanced weighted superposition attraction algorithm," Hacettepe Journal of Mathematics and Statistics, vol. 48, no. 3, pp. 859-882, 2019. 